\title{
The cover time of a biased random walk on a random regular graph of odd degree
}

\author{
Tony Johansson* \\ Department of Mathematics \\ Uppsala University \\ Uppsala, Sweden \\ tony.johansson@math.uu.se
}

Submitted: Nov 19, 2018; Accepted: Nov 6, 2019; Published: Oct 16, 2020

(C) The author. Released under the CC BY-ND license (International 4.0).

\begin{abstract}
We consider a random walk process on graphs introduced by Orenshtein and Shinkar (2014). At any time, the random walk moves from its current position along a previously unvisited edge chosen uniformly at random, if such an edge exists. Otherwise, it walks along a previously visited edge chosen uniformly at random. For the random $r$-regular graph, with $r$ a constant odd integer, we show that this random walk process has asymptotic vertex and edge cover times $\frac{1}{r-2} n \log n$ and $\frac{r}{2(r-2)} n \log n$, respectively, generalizing a result of Cooper, Frieze and the author (2018) from $r=3$ to any odd $r \geqslant 3$. The leading term of the asymptotic vertex cover time is now known for all fixed $r \geqslant 3$, with Berenbrink, Cooper and Friedetzky (2015) having shown that the vertex cover time is asymptotic to $\frac{r n}{2}$ when $r \geqslant 4$ is even.
\end{abstract}

Mathematics Subject Classifications: 05C80, 05C 81

\section{Introduction}

We consider a biased random walk on the random $r$-regular $n$-vertex graph $G_{r}$ for any odd fixed $r \geqslant 5$, i.e. a graph chosen uniformly at random from the set of $r$-regular graph on an even number $n$ of vertices. This random walk was introduced by Orenshtein and Shinkar [10]. In short, the walker walks from its current vertex along a previously unvisited edge whenever possible, and otherwise along an already visited edge chosen uniformly at random. See Section 2 for a precise definition. In [5] it is shown that with

\footnotetext{
${ }^{*}$ Supported in part by the Knut and Alice Wallenberg Foundation.
} 
high probability, $G_{3}$ is such that the expected vertex cover time $C_{V}^{b}\left(G_{3}\right)$ and expected edge cover time $C_{E}^{b}\left(G_{3}\right)$ of the biased random walk satisfy ${ }^{1}$

$$
C_{V}^{b}\left(G_{3}\right) \sim n \log n, \quad C_{E}^{b}\left(G_{3}\right) \sim \frac{3}{2} n \log n .
$$

We generalize this result as follows.

Theorem 1. Suppose $3 \leqslant r=O(1)$ is odd, and let $G_{r}$ be chosen uniformly at random from the set of $r$-regular graphs on $n$ vertices. Then with high probability, $G_{r}$ is such that

$$
C_{V}^{b}\left(G_{r}\right) \sim \frac{1}{r-2} n \log n, \quad C_{E}^{b}\left(G_{r}\right) \sim \frac{r}{2(r-2)} n \log n .
$$

With this the asymptotic leading term of $C_{V}^{b}\left(G_{r}\right)$ is known for all $r \geqslant 3$, with Berenbrink, Cooper and Friedetzky [3] having previously shown that $C_{V}^{b}\left(G_{r}\right) \sim \frac{r n}{2}$ for any even $r \geqslant 4$. They also showed that for even $r, C_{E}^{b}\left(G_{r}\right)=O(\omega n)$ for any $\omega$ tending to infinity with $n$, with the $\omega$ factor owing to the w.h.p. ${ }^{2}$ existence of cycles of length up to $\omega$.

Cooper and Frieze [6] considered the simple random walk on $G_{r}$, showing that for any $r \geqslant 3, C_{V}^{s}\left(G_{r}\right) \sim \frac{r-1}{r-2} n \log n$ and $C_{E}^{s}\left(G_{r}\right) \sim \frac{r(r-1)}{2(r-2)} n \log n$, and we see that the biased random walk speeds up the cover time by a factor of $1 /(r-1)$ for odd $r$. Cooper and Frieze [7] also consider the non-backtracking random walk, i.e. the walk which at no point reuses the edge used in the previous step, showing that $C_{V}^{n b}\left(G_{r}\right) \sim n \log n$ and $C_{E}^{n b}\left(G_{r}\right) \sim \frac{r}{2} n \log n$. Here, the biased random walk gains a factor of $1 /(r-2)$ for odd $r$.

Theorem 1 will follow from the following theorem. Let $C_{V}^{b}(G ; s)\left(C_{E}^{b}(G ; t)\right)$ denote the expected time taken for the biased random walk to visit $s$ vertices ( $t$ edges). Note that $C^{b}(G ; \cdot)$ is defined as an expectation over the space of random walks on the fixed graph $G$, and that $\mathbb{E}\left(C^{b}\left(G_{r} ; \cdot\right)\right)$ takes the expectation of $C^{b}(G ; \cdot)$ when $G$ is chosen uniformly at random from the set of $r$-regular graphs.

Theorem 2. Suppose $r \geqslant 3$ is odd, and suppose $G_{r}$ is chosen uniformly at random from the set of $r$-regular graphs on an even number $n$ of vertices. Let $\left(1-\log ^{-r} n\right) n \leqslant s \leqslant n$ and $\left(1-\log ^{-2} n\right) \frac{r n}{2} \leqslant t \leqslant r n / 2$, and let $\varepsilon>0$. Then

$$
\begin{aligned}
& \mathbb{E}\left(C_{V}^{b}\left(G_{r} ; s\right)\right)=\frac{1 \pm \varepsilon}{r-2} n \log \left(\frac{n}{n-s+1}\right)+o(n \log n), \\
& \mathbb{E}\left(C_{E}^{b}\left(G_{r} ; t\right)\right)=\frac{r \pm \varepsilon}{2(r-2)} n \log \left(\frac{r n}{r n-2 t+1}\right)+o(n \log n) .
\end{aligned}
$$

We take $a=b \pm c$ to mean that $b-c<a<b+c$. The $\left(1-\log ^{-2} n\right)$ factor in the lower bound for $t$ is a fairly arbitrary choice, and may be replaced by $(1-1 / \omega)$ for any $\omega$ tending to infinity sufficiently slowly. The specific choice of $\log ^{-2} n$ is made to aid readability.

Applying Theorem 2 with $s=n$ and $t=r n / 2$ gives $\mathbb{E}\left(C_{V}^{b}\left(G_{r}\right)\right) \sim \frac{1}{r-2} n \log n$ and $\mathbb{E}\left(C_{E}^{b}\left(G_{r}\right)\right) \sim \frac{r}{2(r-2)} n \log n$. Some more work is needed to conclude that w.h.p. $G_{r}$ is such that $C_{V}^{b}\left(G_{r}\right), C_{E}^{b}\left(G_{r}\right)$ have the same asymptotic values, see Section 6.3.

\footnotetext{
${ }^{1}$ We say that $a_{n} \sim b_{n}$ if $\lim a_{n} / b_{n}=1$.

${ }^{2}$ An event $\mathcal{E}$ holds with high probability (w.h.p.) if $\operatorname{Pr}\{\mathcal{E}\} \rightarrow 0$ as $n \rightarrow \infty$.
} 


\section{Proof outline}

The random $r$-regular graph $G_{r}$ is chosen according to the configuration model, introduced by Bollobás [4]. Each vertex $v \in[n]$ is associated with a set $\mathcal{P}(v)$ of $r$ configuration points, and we let $\mathcal{P}=\cup_{v} \mathcal{P}(v)$. We choose u.a.r. (uniformly at random) a perfect matching $\mu$ of the points in $\mathcal{P}$. Each $\mu$ induces a multigraph $G$ on $[n]$ in which $u$ is adjacent to $v$ if and only if $\mu(x) \in \mathcal{P}(v)$ for some $x \in \mathcal{P}(u)$, allowing parallel edges and self-loops. Any simple $r$-regular graph is equally likely to be chosen under this model.

We study a biased random walk. On a fixed graph $G$, this process is defined as follows. Initially, all edges are declared unvisited, and we choose some vertex $v_{0}$ as the active vertex. At any point of the walk, the walk moves from the active vertex $v$ along an edge chosen uniformly at random from the unvisited edges incident to $v$, after which the edge is permanently declared visited. If there are no unvisited edges incident to $v$, the walk moves along a visited edge chosen uniformly at random. The other endpoint of the chosen edge is declared active, and the process is repeated.

A biased random walk on the random $r$-regular graph can be seen as a random walk on the configuration model, where we expose $\mu$ along with the walk as follows. Initially choosing some point $x_{0} \in \mathcal{P}$, we walk to $x_{1}=\mu\left(x_{0}\right)$, chosen u.a.r. from $\mathcal{P} \backslash\left\{x_{0}\right\}$. Suppose $x_{1} \in \mathcal{P}\left(v_{1}\right)$. From $x_{1}$, the walk moves to some unvisited $x_{2} \in \mathcal{P}\left(v_{1}\right)$. In general, if $W_{k}=\left(x_{0}, x_{1}, \ldots, x_{k}\right)$ then (i) if $k$ is odd, the walk moves to $x_{k+1}=\mu\left(x_{k}\right)$ (chosen u.a.r. from $\mathcal{P} \backslash\left\{x_{0}, \ldots, x_{k}\right\}$ if $x_{k}$ is previously unvisited), and (ii) if $k$ is even, the walk moves from $x_{k} \in \mathcal{P}\left(v_{k}\right)$ to $x_{k+1} \in \mathcal{P}\left(v_{k}\right)$, chosen u.a.r. from the unvisited points of $\mathcal{P}\left(v_{k}\right)$ if such exist, otherwise chosen u.a.r. from all of $\mathcal{P}\left(v_{k}\right)$.

We define $C(t)$ to be the number of steps taken immediately before the walk exposes its $t$-th distinct edge. To be precise, if $W_{k}=\left(x_{0}, \ldots, x_{k}\right)$ denotes the walk after $k$ steps, then

$$
C(t)=\frac{1}{2} \min \left\{k:\left|\left\{x_{0}, x_{1}, \ldots, x_{k}\right\}\right|=2 t-1\right\} .
$$

Note that the set in this definition always will consist of exactly one $k$, which is even. We divide by 2 because the sequence $\left(x_{0}, x_{1}, \ldots, x_{k}\right)$ consists of $\lceil k / 2\rceil$ edge traversals and $\lfloor k / 2\rfloor$ steps between configuration points within one vertex, and the quantity we are interested in is the number of edge traversals. We also let $W(t)=W_{2 C(t)}$. Note that $C(t)$ is a random variable over the combined probability space of random graphs and random walks, as opposed to the classical cover times $C_{V}^{b}\left(G_{r}\right)$ and $C_{E}^{b}\left(G_{r}\right)$ which are variables over the space of random graphs only.

We will show (Lemma 13) that if $t_{1}=\left(1-\log ^{-1 / 2} n\right) \frac{r n}{2}$ then

$$
\mathbb{E}\left(C\left(t_{1}\right)\right)=o(n \log n),
$$

which does not contribute significantly to the cover time. The main part of the proof is devoted to calculating $\mathbb{E}(C(t+1)-C(t))$ when $t \geqslant t_{1}$. We define the random graph $G(t) \subseteq G_{r}$ as the graph spanned by the first $t$ distinct edges visited by the walk, i.e. the trace of $W(t)$. If, immediately after discovering its $t$-th edge, the biased random walk inhabits a vertex incident to no unvisited edges, then a simple random walk commences 
on $G(t)$, and $C(t+1)-C(t)$ is the number of steps taken for this simple random walk to hit a vertex incident to an unvisited edge.

We construct from $G(t)$ a graph $G^{*}(t)$ by contracting all vertices incident to at least one unvisited edge into one "supervertex" $x$. Thus, conditioning on $W(t)$, the graph $G^{*}(t)$ is a fixed graph, i.e. one with no random edges. We will show that when $t \geqslant t_{1}$, w.h.p. $x$ lies on "few" cycles of "short" length and has the appropriate number of self-loops (to be made precise in Section 4), which will imply that the expected hitting time of $x$ for a simple random walk on $G^{*}(t)$ is

$$
\mathbb{E}(H(x)) \sim \frac{1}{r-2} \frac{r n}{r n-2 t} .
$$

Readers familiar with the proof for the cubic graph [5] will recognize the general idea of this outline. In the case of cubic graphs, the set of vertices visited exactly once coincides with the set of vertices incident to one unvisited edge, modulo the starting vertex of the walk. This is no longer true when $r \geqslant 5$, which forces a more detailed study of the edges not visited by the walk.

The paper is laid out as follows. Sections 3, 4 and 5 respectively discuss properties of the random regular graph, hitting times of simple random walks, and a uniformity lemma for biased random walks, and may be read in any order. Section 6 contains the calculation of the cover time. Sections 7 and 8 are devoted to bounding the sizes of certain sets appearing in the calculations.

\section{Properties of the random regular graph}

Here we collect some properties of random $r$-regular graphs, chosen according to the configuration model.

Lemma 3. Let $r \geqslant 3$. Let $G_{r}$ denote the random $r$-regular graph on vertex set $[n]$, chosen according to the configuration model. Let $\omega$ tend to infinity arbitrarily slowly with $n$. Its value will always be small enough so that where necessary, it is dominated by other quantities that also go to infinity with $n$.

(i) With high probability, the second largest in absolute value of the eigenvalues of the transition matrix for a simple random walk on $G_{r}$ is at most 0.99 .

(ii) With high probability, $G_{r}$ contains at most $\omega r^{\omega}$ cycles of length at most $\omega$,

(iii) The probability that $G_{r}$ is simple is $\Omega(1)$.

Friedman [8] showed that for any $\varepsilon>0$, the second eigenvalue of the transition matrix is at most $2 \sqrt{r-1} / r+\varepsilon$ w.h.p., which gives (i). Property (ii) follows from the Markov inequality, given that the expected number of cycles of length $k \leqslant \omega$ can be bounded by $O\left(r^{k}\right)$. For the proof of (iii) see Frieze and Karoński [9], Theorem 10.3. Note that (iii) implies that any property which holds w.h.p. for the configuration multigraph holds w.h.p. for simple $r$-regular graphs chosen uniformly at random. 
Let $G(t)$ denote the random graph formed by the edges visited by $W(t)$. Let $X_{i}(t)$ denote the set of vertices incident to $i$ unvisited edges in $G(t)$ for $i=0,1, \ldots, r$. Let $\bar{X}(t)=X_{1}(t) \cup \cdots \cup X_{r}(t)$ denote the set of vertices incident to at least one unvisited edge. Let $G^{*}(t)$ denote the graph obtained from $G(t)$ by contracting the set $\bar{X}(t)$ into a single vertex, retaining all edges. Define $\lambda^{*}(t)$ to be the second largest eigenvalue of the transition matrix for a simple random walk on $G^{*}(t)$.

We note that by [2, Corollary 3.27], if $\Gamma$ is a graph obtained from $G$ by contracting a set of vertices, retaining all edges, then $\lambda(\Gamma) \leqslant \lambda(G)$. This implies that $\lambda^{*}(t)=\lambda\left(G^{*}(t)\right) \leqslant$ $\lambda(G) \leqslant 0.99$ for all $t$. Initially, for small $t$, we find that w.h.p. $G^{*}(t)$ consists of a single vertex. In this case there is no second eigenvalue and we take $\lambda^{*}(t)=0$. This is in line with the fact that a random walk on a one vertex graph is always in the steady state.

We define $C(t)$ to be the number of steps the biased random walk takes to traverse $t$ distinct edges of $G_{r}$. Of course, if $G_{r}$ is disconnected and the random walk starts in a connected component of less than $t$ edges, then $C(t)=\infty$. We resolve this by defining a stopping time $T^{*}=\min \left\{t: \lambda^{*}(t)>0.99\right\}$, and setting $C^{*}(t)=C(\min \{t, T\})$. Strictly speaking, the estimates of $C(t)$ in the upcoming sections are estimates of $C^{*}(t)$, but we do not make any explicit distinction between the two, noting that by Lemma 3 (i), w.h.p. $T^{*}=\infty$ which implies that $C^{*}(t)=C(t)$ for all $t$.

\section{Simple random walks}

We are interested in calculating $C(t+1)-C(t)$, i.e. the time taken between discovering the $t$-th and the $(t+1)$-th edge. Between the two discoveries, the biased random walk can be coupled to a simple random walk on the graph induced by $W(t)$, and in this section we derive the hitting time of a certain type of expanding vertex set.

Implicitly, when we state results about a vertex set $S$ in a graph $G$ we are considering a sequence of vertex sets $\left(S_{n}\right)$ in a graph sequence $\left(G_{n}\right)$. We say that $G$ has positive eigenvalue gap if the second largest eigenvalue $\lambda_{n}$ of the transition matrix for $G_{n}$ satisfies $\lim \sup \lambda_{n}<1$.

Consider a simple random walk on an $r$-regular graph $G=(V, E)$ with eigenvalue gap $1-\lambda>0$. For a set $S$ of vertices and a probability measure $\rho$ on $V$, let $\mathbb{E}_{\rho}(H(S))$ denote the expected hitting time of the set $S$, i.e. the number of steps the walk takes until it reaches $S$, when the initial vertex is chosen according to $\rho$. Let $\pi$ denote the stationary distribution of the random walk, uniform in the case of a regular graph and proportional to degrees in general. Let $P_{u}^{(t)}(v)$ denote the probability that a simple random walk starting at $u$ occupies vertex $v$ at time $t$.

Lemma 4. Suppose $v$ is a vertex of a graph. Then the hitting time of $v$, starting from the stationary distribution $\pi$, is given by

$$
\mathbb{E}_{\pi}(H(v))=\frac{Z_{v v}}{\pi_{v}}
$$


where

$$
Z_{v v}=\sum_{t \geqslant 0}\left(P_{v}^{(t)}(v)-\pi_{v}\right)
$$

Lemma 4 can be found in [2] (Lemma 2.11), and can be applied to hitting times of sets by contracting a set of vertices to a single vertex, retaining all edges. The following bound will be frequently used. Suppose $G$ is a graph with eigenvalue gap $1-\lambda(G)$, and $S$ is a set of vertices in $G$. Then if $G_{S}$ is the graph obtained by contracting $S$ into a single vertex, retaining all edges, we have equal hitting times for $S$ in $G$ and $G_{S}$ and

$$
\begin{aligned}
\mathbb{E}_{\pi}(H(S))=\frac{n}{|S|} \sum_{t \geqslant 0}\left(P_{S}^{(t)}(S)-\pi_{S}\right) & \leqslant \frac{n}{|S|} \sum_{t \geqslant 0} \lambda\left(G_{S}\right)^{t} \\
& =\frac{1}{1-\lambda\left(G_{S}\right)} \frac{n}{|S|} \leqslant \frac{1}{1-\lambda(G)} \frac{n}{|S|}
\end{aligned}
$$

Indeed, $\left|P_{u}^{(t)}(v)-\pi_{v}\right| \leqslant(d(v) / d(u))^{1 / 2} \lambda^{t}$ for any $u, v, t$ in a graph with eigenvalue gap $1-\lambda$ (see for example Jerrum and Sinclair [11]), and $\lambda(\Gamma) \leqslant \lambda(G)$ for any $\Gamma$ obtained from $G$ by contracting a set of vertices (see [2, Corollary 3.27]). From an arbitrary starting distribution $\rho$, we have for any constant $\varepsilon>0$,

$$
\mathbb{E}_{\rho}(H(S)) \leqslant O(\log n)+(1+\varepsilon) \mathbb{E}_{\pi}(H(S))=O\left(\log n+\frac{n}{|S|}\right)
$$

which follows from the fact that if the graph has positive eigenvalue gap, after $O(\log n)$ steps the walk has mixed to within $\varepsilon$ distance in total variation from the stationary distribution.

In the following lemma we define $N_{d}(S)$ to be the set of vertices at distance exactly $d$ from the vertex set $S$.

Lemma 5. Suppose $G$ is an $r$-regular graph on $n$ vertices with positive eigenvalue gap. Let $\omega$ tend to infinity arbitrarily slowly with $n$. Suppose $S$ is a set of vertices with $|S|=o(n)$ even such that

$$
\left|N_{d}(S)\right|=(r-1)^{d}|S|
$$

for all $1 \leqslant d \leqslant \omega$. Then

$$
\mathbb{E}_{\pi}(H(S)) \sim \frac{r}{r-2} \frac{n}{|S|}
$$

Proof. We first note that the set $S$ contains exactly $|S| / 2$ edges. Indeed, as $|N(S)|=$ $(r-1)|S|$ and the total degree of $S$ is $r|S|, S$ contains at most $|S| / 2$ edges. As $\left|N_{2}(S)\right|=$ $(r-1)^{2}|S|$, each vertex of $N(S)$ must have exactly one edge to $S$, implying that $S$ contains at least $|S| / 2$ edges.

Consider the graph $G_{S}$ obtained by contracting $S$ into a single node $s$, retaining all edges. In the graph $G_{S}, s$ has degree $r|S|$. Then $s$ is a node with exactly $|S| / 2$ self-loops, and is otherwise contained in no cycle of length at most $\omega$, as $\left|N_{d}(S)\right|=(r-1)^{d}|S|$ ensures 
that $G_{S}$ is locally a tree up to distance $\omega$ from $s$. Since $\pi_{s}=|S| / n=o(1)$ we may choose $\omega$ tending to infinity with $\omega \pi_{s}=o(1)$. We have

$$
Z_{s s}=\sum_{t \geqslant 0}\left(P_{s}^{(t)}(s)-\pi_{s}\right)=\left[\sum_{t=0}^{\omega} P_{s}^{(t)}(s)\right]-o(1)+\sum_{t>\omega}\left|P_{s}^{(t)}(s)-\pi_{s}\right| .
$$

Repeating the argument following (1),

$$
\sum_{t>\omega}\left|P_{s}^{(t)}(s)-\pi_{s}\right| \leqslant \sum_{t>\omega} \lambda^{t}=O\left(\lambda^{\omega}\right)=o(1) .
$$

We now argue that

$$
\sum_{t=0}^{\omega} P_{s}^{(t)}(s)=\frac{r}{r-2}+o(1)
$$

It is argued in Cooper and Frieze [6], Lemma 7, that with no loop at vertex $s$, the expected number of returns to $s$ within $\omega$ steps is $(r-1) /(r-2)+o(1)$. With $|S| / 2$ loops, when at $s$, there is a $1 / r$ chance of using the loop and so each visit to $s$ yields $1 /(r-1)$ expected returns. I.e. the 2 of [6] becomes

$$
\frac{r-1}{r-2} \times\left(1+\frac{1}{r-1}\right)=\frac{r}{r-2} .
$$

Definition 6. Let $G=(V, E)$ be an $r$-regular graph. A set $S \subseteq V$ is a root set of order $\ell$ if (i) $|S| \geqslant \ell^{5}$, (ii) the number of edges with both endpoints in $S$ is between $|S| / 2$ and $\left(1 / 2+\ell^{-3}\right)|S|$, and (iii) there are at most $|S| / \ell^{3}$ paths of length at most $\ell$ between vertices of $S$ which use no edges induced by $S$.

The set $S$ in Lemma 5 can be thought of as a "pure" root set, and we now show that the hitting time remains similar when we allow some impurities in both the structure of the set and in the starting distribution.

Lemma 7. Let $\omega$ tend to infinity arbitrarily slowly with $n$. Suppose $G$ is an $r$-regular graph on $n$ vertices whose transition matrix has second largest eigenvalue $\lambda \leqslant 0.99$, containing at most $\omega r^{\omega}$ cycles of length at most $\omega$. Suppose $S$ is a root set of order $\omega$, and a simple random walk is initated at a vertex $y$ chosen according to a distribution $\mu$ satisfying $\mu(y) \leqslant r /|S|$ for $y \in S$ and $\mu(y)=0$ for $y \notin S$. The expected time to reach a vertex in $S$ at distance at least 2 from $y$ is then

$$
\left(\frac{r}{r-2} \pm \varepsilon\right) \frac{n}{|S|}+O(\log n)
$$

for any constant $\varepsilon>0$.

Proof. We first argue that

$$
\mathbb{E}_{\pi}(H(S)) \sim \frac{r}{r-2} \frac{n}{|S|}
$$


Consider the contracted graph $G_{S}$, and let $s$ denote the contracted node. Then $s$ has degree $r|S|$, and $s$ has at most $\left(1 / 2+\omega^{-3}\right)|S|$ self-loops. Apart from the self-loops, $s$ lies on at most $|S| / \omega^{3}$ cycles of length at most $\omega$, as any cycle of $G_{S}$ containing $s$ corresponds to a path between members of $S$ in $G$.

Let $R=N(S)$, and note that $|R|=\Omega(|S|)$. Consider the graph $\Gamma$, defined as $G_{S}$ induced on the set of vertices at distance $1,2, \ldots, \omega$ from $s$. Note that $s$ is not included in $\Gamma$. The graph $\Gamma$ contains all of $R$, and as $s$ lies on at most $|S| / \omega^{3}$ cycles of length at most $\omega$ in $G_{S}$, the number of components in $\Gamma$ containing more than one member of $R$ is $O\left(|S| / \omega^{3}\right)=O\left(|R| / \omega^{3}\right)$. As $G$ contains at most $\omega r^{\omega}$ short cycles, the number of components of $\Gamma$ containing a cycle is at most $\omega r^{\omega}=O\left(|R| / \omega^{3}\right)$ (choosing $\omega$ tending to infinity sufficiently slowly). This leaves $(1-o(1))|R|$ connected components in $\Gamma$ which are all complete $(r-1)$-ary trees of height $\omega$, each rooted at a member of $R$ and containing no other member of $R$. Let $T$ denote the set of vertices on such components.

Arbitrarily choose $|S| / 2$ of the self-loops of $s$ in $G_{S}$, and designate them as good. Also say that an edge is good if it has both endpoints in $T \cup\{s\}$. All other edges are bad.

Consider a simple random walk $Z(\tau)$ of length $\omega$ on $G_{S}$, starting at $s$. Let $\mathcal{B}_{\tau}$ denote the event that $Z(\tau)$ traverses a bad edge to reach $Z(\tau+1)$. Whenever the walk visits $s$, the probability that it chooses a bad edge is $O\left(\omega^{-3}\right)$. If the walk is inside $T$, there are no bad edges to choose. So for any $\tau \geqslant 0$ we have

$$
\begin{aligned}
P_{s}^{(\tau)}(s) & =\operatorname{Pr}\left\{Z(\tau)=s \cap \bigcap_{r=0}^{\tau-1} \overline{\mathcal{B}_{r}}\right\}+\operatorname{Pr}\left\{Z(\tau)=s \cap \bigcup_{r=0}^{\tau-1} \mathcal{B}_{r}\right\} \\
& =\operatorname{Pr}\left\{Z(\tau)=s \cap \bigcap_{r=0}^{\tau-1} \overline{\mathcal{B}_{r}}\right\}+O\left(\omega^{-2}\right) .
\end{aligned}
$$

If $\mathcal{B}_{r}$ does not occur for any $r \leqslant \tau-1$, then the walk $(Z(0), \ldots, Z(\tau-1))$ can be viewed as the same Markov chain as considered in Lemma 5. So, by Lemma 5,

$$
\sum_{\tau=0}^{\omega} P_{s}^{(\tau)}(s)=\frac{r}{r-2}+O\left(\omega^{-1}\right)
$$

This shows that $\mathbb{E}_{\pi}(H(S)) \sim \frac{r}{r-2} \frac{n}{|S|}$.

We show (3), noting that we only need to consider $|S|=o(n / \log n)$ due to the $O(\log n)$ term. Suppose $y \in S$ is chosen according to a distribution $\mu$ with $\mu(y) \leqslant r /|S|$ for any $y \in S$. Let $S(y)$ denote the set of vertices in $S$ at distance at least 2 from $y$, and let $H$ be the random time at which $S(y)$ is hit. The probability of $H \leqslant \omega$ steps is then bounded by $r / \omega^{3}$, as there are at most $|S| / \omega^{3}$ paths of length at most $\omega$ between vertices of $S$ which use no edges induced by $S$. For any $t$ we have

$$
\left|P_{S}^{(t)}(S)-\pi_{S}\right| \leqslant \lambda^{t},
$$

so if we let $p_{t}$ denote the probability that the walk is in $S$ at time $t$,

$$
\operatorname{Pr}\{H \leqslant t\} \leqslant \operatorname{Pr}\{H<\omega\}+\sum_{s=\omega}^{t} p_{s} \leqslant \frac{r}{\omega^{3}}+\sum_{s=\omega}^{t} \pi_{S}+\lambda^{s}=o(1)+\frac{t|S|}{n} .
$$


Since $|S|=o(n / \log n)$, we conclude that $\operatorname{Pr}\{H \leqslant C \log n\}=o(1)$ for any constant $C$. With $t=C \log n$ for $C$ large enough, we have $P_{S}^{(t)}(\cdot)$ within $\varepsilon$ of $\pi$ in total variation distance. If $H^{\prime}$ denotes the first time after time $t$ that $S(y)$ is hit, we then have

$$
\mathbb{E}\left(H^{\prime}\right)=t+(1 \pm \varepsilon) \mathbb{E}_{\pi}(H(S(y)))=O(\log n)+\left(\frac{r}{r-2} \pm \varepsilon\right) \frac{n}{|S|} .
$$

Since $H^{\prime}=H$ with probability $1-o(1)$, the lemma follows.

The next lemma is needed in the study of the sizes of $X_{i}(t)$, and will be applied with $R=X_{1}(t), S=\bar{X}(t)$.

Lemma 8. Let $G$ be an r-regular graph with positive eigenvalue gap. Let $R \subseteq S \subseteq V$ be vertex sets. Suppose a simple random walk is initiated at a vertex y chosen according to a distribution $\mu$ with $\mu(y) \leqslant \frac{r}{|R|}$ for $y \in R$ and $\mu(y)=0$ for $y \notin R$. Suppose the walk ends as soon as it hits $S \backslash\{y\}$. Then there is a constant $B>0$ such that for any $x \in S$, the probability that the walk ends at $x$ is at most $B /|R|$.

Proof. We will in general write $\left(w_{0}, w_{1}, \ldots\right)$ for a simple random walk. Define $S(x, y)=$ $S \backslash\{x, y\}$. The following reversibility property is central to the proof. If $T_{S(x, y)}$ denotes the first time at which $S(x, y)$ is visited, then as the graph is regular,

$$
P_{x}\left(w_{t}=y \text { and } T_{S(x, y)}>t\right)=P_{y}\left(w_{t}=x \text { and } T_{S(x, y)}>t\right), \quad \text { for all } t \geqslant 0 .
$$

Let $\mu$ denote the uniform probability measure on $R$. Then, writing $\cdot$ or $y$ for the starting vertex chosen by $\mu$, the probability that $x$ is the first vertex of $S \backslash\{y\}$ to be hit is

$$
\begin{aligned}
P_{\mu}\left(T_{x}<T_{S(x, \cdot)}\right) & =\sum_{t \geqslant 0} P_{\mu}\left(t=T_{x} \text { and } T_{S(x, \cdot)}>t\right) \\
& \leqslant \sum_{t \geqslant 0} P_{\mu}\left(w_{t}=x \text { and } T_{S(x, \cdot)}>t\right) \\
& \leqslant \frac{r}{|R|} \sum_{t \geqslant 0} \sum_{y \in R} P_{y}\left(w_{t}=x \text { and } T_{S(x, y)}>t\right) \\
& =\frac{r}{|R|} \sum_{y \in R} \sum_{t \geqslant 0} P_{x}\left(w_{t}=y \text { and } T_{S(x, y)}>t\right) .
\end{aligned}
$$

Write

$$
Q_{x y}(t)=P_{x}\left(w_{t}=y \text { and } w_{1}, \ldots, w_{t-1} \neq y \text { and } T_{S(x, y)}>t\right),
$$

so that $Q_{x y}(t)$ denotes the probability that the walk avoids $S(x, y)$ and first visits $y$ is at time $t$ (or returns to $y$ if $x=y$ ). Then for all $t \geqslant 1$,

$$
P_{x}\left(w_{t}=y \text { and } T_{S(x, y)}>t\right)=\sum_{s=1}^{t} Q_{x y}(s) P_{y}\left(w_{t-s}=y \text { and } T_{S(x, y)}>t-s\right),
$$


so, letting $\mathbf{1}_{x=y}=1$ if $x=y$ and 0 otherwise,

$$
\begin{aligned}
\sum_{t \geqslant 0} P_{x}\left(w_{t}=y \text { and } T_{S(x, y)}>t\right) & =\mathbf{1}_{x=y}+\sum_{t \geqslant 1} \sum_{s=1}^{t} Q_{x y}(s) P_{y}\left(w_{t-s}=y \text { and } T_{S(x, y)}>t-s\right) \\
& =\mathbf{1}_{x=y}+\left[\sum_{s \geqslant 1} Q_{x y}(s)\right]\left[\sum_{t \geqslant 0} P_{y}\left(w_{t}=y \text { and } T_{S(x, y)}>t\right)\right] .
\end{aligned}
$$

As hitting times are almost surely finite, writing $T_{y}^{+}=\min \left\{t \geqslant 1: w_{t}=y\right\}$, we have

$$
\sum_{s \geqslant 1} Q_{x y}(s)=P_{x}\left(T_{y}^{+}<T_{S(x, y)}\right)
$$

Letting $x=y$ and plugging (6) into (5) we obtain

$$
\sum_{t \geqslant 0} P_{y}\left(w_{t}=y \text { and } T_{S(x, y)}>t\right)=\frac{1}{1-\sum_{s \geqslant 1} Q_{y y}(s)}=\frac{1}{P_{y}\left(T_{S(x, y)}<T_{y}^{+}\right)} .
$$

Now let $x \neq y$ and note that in this case $T_{y}^{+}=T_{y}$. Plug (6) and (7) into (5) to obtain

$$
\sum_{t \geqslant 0} P_{x}\left(w_{t}=y \text { and } T_{S(x, y)}>t\right)=\frac{P_{x}\left(T_{y}<T_{S(x, y)}\right)}{P_{y}\left(T_{S(x, y)}<T_{y}^{+}\right)} .
$$

The identity (8) also holds for $x=y$, as $P_{y}\left(T_{y}<T_{S(x, y)}\right)=1$, matching the expression in (7). Plugging (8) into (4) yields

$$
P_{\mu}\left(T_{x}<T_{S(x, y)}\right)=\frac{r}{|R|} \sum_{y \in R} \frac{P_{x}\left(T_{y}<T_{S(x, y)}\right)}{P_{y}\left(T_{S(x, y)}<T_{y}^{+}\right)} .
$$

Claim 9.

$$
P_{y}\left(T_{S(x, y)}<T_{y}^{+}\right) \geqslant \frac{1-\lambda}{2} .
$$

Proof of Claim 9. Corollary 2.8 of [2] states that for any states $i, j$ in a Markov chain,

$$
P_{i}\left(T_{j}<T_{i}^{+}\right)=\frac{1}{\pi_{i}\left(\mathbb{E}_{i}\left(T_{j}\right)+\mathbb{E}_{j}\left(T_{i}\right)\right)} .
$$

We consider running a simple random walk on the graph obtained by contracting $S(x, y)$ to a single vertex, and set $i=y, j=S(x, y)$. As $\pi_{y}=1 / n$, we have

$$
P_{y}\left(T_{S(x, y)}<T_{y}^{+}\right)=\frac{n}{\mathbb{E}_{y}\left(T_{S(x, y)}\right)+\mathbb{E}_{S(x, y)}\left(T_{y}\right)} .
$$

For any two states $k, \ell$ in a Markov chain with eigenvalue gap $1-\lambda$ we have

so

$$
\mathbb{E}_{k}\left(T_{\ell}\right) \leqslant O(\log n)+\frac{1}{1-\lambda} \frac{1}{\pi_{\ell}}
$$

$$
P_{y}\left(T_{S}<T_{y}^{+}\right) \geqslant \frac{n}{O(\log n)+\frac{1}{1-\lambda} \frac{n}{|S(x, y)|}+\frac{n}{1-\lambda}} \geqslant \frac{1-\lambda}{2} .
$$


Applying Claim 9 to (9) and choosing $B=\frac{2 r}{1-\lambda}$ finishes the proof, as the events $\left\{T_{y}<T_{S(x, y)}\right\}$ are mutually exclusive for $y \in R$, so

$$
P_{\mu}\left(T_{x}<T_{S(x, y)}\right) \leqslant \frac{2 r}{(1-\lambda)|R|} \sum_{y \in R} P_{x}\left(T_{y}<T_{S(x, y)}\right) \leqslant \frac{B}{|R|} .
$$

\section{On vertices with at least one unvisited edge}

We now move from considering simple random walks, to the biased random walk $W_{k}$. Recall that $W(t)=W_{2 C(t)}$ denote the state of the walk immedately after it visits its $(2 t-1)$-th distinct configuration point. See Section 2 for details.

The walk $W(t)$ induces a colouring on the edges and vertices of $G_{r}$ as follows. An edge is coloured red, green or blue if it has been visited zero, one or at least two time(s), respectively. A vertex is (i) green if it is incident to exactly $r-1$ green edges and one red edge, (ii) red if it is incident to red edges only, and (iii) blue otherwise.

Recall that $X_{i}(t)$ denotes the set of vertices incident to exactly $i$ red edges in $W(t)$. We let $X_{1}^{g}(t), X_{1}^{b}(t)$ denote the green and blue vertices of $X_{1}(t)$, respectively, and set

$$
Z(t)=X_{1}^{b}(t) \cup \bigcup_{i=2}^{r} X_{i}(t) .
$$

We have $\bar{X}(t)=X_{1}^{g}(t) \cup Z(t)$. Note that the number of configuration points not visited by $W(t)$ is exactly $r n-2 t+1$. We will eventually show that $\left|X_{1}^{g}(t)\right|=(r n-2 t)(1-o(1))$ and $|Z(t)|=o(r n-2 t)$, so that $X_{1}^{g}(t)$ makes up almost all of $\bar{X}(t)$ when $t=(1-\delta) \frac{r n}{2}$ for some $\delta=o(1)$. In this section we present a "sprinkling" tool used to show that $X_{1}^{g}(t)$ is a root set of order $\omega$, which will imply that $\bar{X}(t)$ is also a root set of order $\omega$. Before this, we state our results on the necessary set sizes.

\subsection{Set sizes}

Define

$$
\delta_{0}=\frac{1}{\log \log n}, \delta_{1}=\frac{1}{\log ^{1 / 2} n}, \delta_{2}=\frac{1}{\log ^{2} n}, \delta_{3}=n^{-3 / 4}, \delta_{4}=n^{-1} \log n,
$$

and $t_{i}=\left(1-\delta_{i}\right) \frac{r n}{2}$ for $0 \leqslant i \leqslant 4$. From this point on we will use $t$ and $\delta$ interchangeably to denote time, and the two are always related by $t=(1-\delta) \frac{r n}{2}$.

Lemma 10. Let $0<\varepsilon<r-2$. Then

(i) for any fixed $t \geqslant t_{1}$, w.h.p. the number of green vertices in $X_{1}(t)$ is

$$
X_{1}^{g}(t)=r n \delta(1-o(1)),
$$

(ii) for any fixed $t_{1} \leqslant t \leqslant t_{3}$, w.h.p. the number of green edges $\Phi(t)$ satisfies

$$
\Phi(t) \geqslant n \delta^{1 / 2}
$$


(iii) for any fixed $t \geqslant t_{1}$, w.h.p. the size of $Z(t)$ is

$$
Z(t)=O\left(n \delta^{3 / 2}\right)
$$

(iv) for any fixed $t \geqslant t_{1}$ such that $n \delta^{r / 2} \rightarrow \infty$ or $n \delta^{r / 2} \rightarrow 0$, w.h.p. the number of unvisited vertices is

$$
X_{r}(t)=n \delta^{r / 2}(1+o(1))
$$

Parts (i), (iii) and (iv) are proved in Section 7, and part (ii) in Section 8.

\subsection{A sprinkling technique}

The green edges and vertices are the focus of this section. Suppose $W=W(t)=$ $\left(x_{0}, x_{1}, \ldots, x_{k}\right)$. Recall that a vertex is green if $r-1$ of its configuration points appear exactly once in $W$, and its remaining configuration point does not. Suppose $x_{2 i}, x_{2 i+1}, x_{2 i+2}$, $x_{2 i+3}$ are consecutive configuration points visited exactly once by $W$, where $x_{2 i+1}, x_{2 i+2}$ belong to a green vertex $v$. In this situation we call the pair $\left(x_{2 i+1}, x_{2 i+2}\right)$ a green link. Let $L(W) \subseteq \mathcal{P} \times \mathcal{P}$ be the set of green links in $W$. For $i<j$, say that $\left(x_{2 i}, \ldots, x_{2 j+1}\right)$ is a green segment if $\left(x_{2 i+1}, x_{2 i+2}\right),\left(x_{2 i+3}, x_{2 i+4}\right), \ldots,\left(x_{2 j-1}, x_{2 j}\right) \in L(W)$, and a maximal green segment if $\left(x_{2 i-1}, x_{2 i}\right),\left(x_{2 j+1}, x_{2 j+2}\right) \notin L(W)$ (or if $i=0$ or $2 j+1=k$, respectively). Form the contracted walk $\langle W\rangle$ by replacing each maximal green segment $\left(x_{2 i}, \ldots, x_{2 j+1}\right)$ by the single edge $\left(x_{2 i}, x_{2 j+1}\right)$. Two walks $W_{1}, W_{2}$ are said to be equivalent if $\left\langle W_{1}\right\rangle=\left\langle W_{2}\right\rangle$ and $L\left(W_{1}\right)=L\left(W_{2}\right)$, and we let $[W]=(\langle W\rangle, L)$ denote the equivalence class of the walk $W$. Note that $X_{i}\left(W_{1}\right)=X_{i}\left(W_{2}\right)$ for any $i$ and $W_{1} \sim W_{2}$, and that the two walks induce the same vertex colouring.

Lemma 11. If $W$ is such that $\operatorname{Pr}\{[W(t)]=[W]\}>0$, then

$$
\operatorname{Pr}\{W(t)=W \mid[W(t)]=[W]\}=\frac{1}{|[W]|} .
$$

Proof. Recall the distinction between $W_{k}$ and $W(t)$ from Section 2. Let $w=\left(x_{0}, \ldots, x_{k}\right)$ be a walk with $\operatorname{Pr}\{W(t)=w\}>0$, i.e. a walk with $\operatorname{Pr}\left\{W_{k}=w\right\}>0$, and such that $\left|\left\{x_{0}, \ldots, x_{k}\right\}\right|=2 t-1$. We calculate $\operatorname{Pr}\left\{W_{k}=w\right\}$ exactly. For $0 \leqslant i<k$, let $w_{i}=$ $\left(x_{0}, \ldots, x_{i}\right)$ and let $s_{i}$ denote the number of distinct edges traversed by $w_{i}$.

If $i$ is even, the walk proceeds to $\mu\left(x_{i}\right)$, i.e. the other endpoint of the edge attached to $x_{i}$. If $x_{i} \notin w_{i-1}$ is visited, we have $\operatorname{Pr}\left\{W_{i+1}=w_{i+1} \mid W_{i}=w_{i}\right\}=1 /\left(r n-2 s_{i}-\right.$ 1). Note that $x_{i+1} \notin w_{i}$ is guaranteed by the assumption that $\operatorname{Pr}\left\{W_{k}=w\right\}>0$. If this is not the first time $x_{i}$ is visited, then $\mu\left(x_{i}\right)$ is uniquely determined by $w_{i}$, and $\operatorname{Pr}\left\{W_{i+1}=w_{i+1} \mid W_{i}=w_{i}\right\}=1$, again using the assumption that $\operatorname{Pr}\left\{W_{k}=w\right\}>0$.

If $i$ is odd, suppose the configuration point $x_{i}$ belongs to a vertex $v$ such that $0 \leqslant$ $j<r$ of the points in $\mathcal{P}(v)$ have not been visited by $w_{i}$. If $j>0$ then we have $\operatorname{Pr}\left\{W_{i+1}=w_{i+1} \mid W_{i}=w_{i}\right\}=1 / j$, while if $j=0$ then this probability is $1 / r$. Say that the step has type $a$ if the probability in question is $1 / a$ (note that the $j=0$ case is type $r$, which will be convenient notationally). 
All in all, letting $e(w)=t-1$ denote the number of distinct edges visited by $w$,

$$
\operatorname{Pr}\left\{W_{k}=w\right\}=\frac{1}{r n} \prod_{a=1}^{r} a^{-i_{a}} \prod_{s=0}^{e(w)-1} \frac{1}{r n-2 s-1},
$$

for some integers $i_{1}, i_{2}, \ldots, i_{r} \geqslant 0$, counting the number of steps of the different types. The $1 / r n$ factor accounts for the starting point of the walk. We argue that (11) can be expressed as a function of $[w]$.

Suppose $w$ has $\langle w\rangle=\left(x_{\langle 0\rangle}, \ldots, x_{\langle\ell\rangle}\right)$ and green link set $L(w)$. If $e(w)$ denotes the number of distinct edges visited by $w$, we have

$$
e(w)=e(\langle w\rangle)+|L(w)| .
$$

It remains to argue that $\prod_{a=1}^{r} a^{-i_{a}}$ is determined by $\langle w\rangle$ and $L(w)$. For a single vertex $v$ let $i_{a}(v)$ denote the number of steps $\left(x_{2 i+1}, x_{2 i+2}\right)$ of type $a$ with $x_{2 i+1} \in \mathcal{P}(v)$. If $v$ is green in the colouring induced by $w$, then $i_{a}(v)=1$ for $a \in\{2,4, \ldots, r-1\}$ and $i_{a}(v)=0$ otherwise. Indeed, $w$ makes exactly $(r-1) / 2$ visits to $v$, and the $i$-th visit involves a step of type $r-2 i+1$. If $v$ is not green, then every visit to a configuration point of $v$ is recorded by $\langle w\rangle$. Indeed, $\langle w\rangle$ is exactly the subsequence of $w$ of configuration points belonging to non-green vertices. So $i_{a}(v)$ is determined by $L(w)$ if $v$ is green and by $\langle w\rangle$ otherwise. This shows that the right-hand side of (11) can be expressed as a function of $[w]$, which finishes the proof.

Conditioning on $[W(t)]=(\langle W(t)\rangle, L)$, we generate $W(t)$ as follows. Suppose $\langle W(t)\rangle=$ $\left(x_{\langle 0\rangle}, x_{\langle 1\rangle}, \ldots, x_{\langle k\rangle}\right)$ is the contracted walk with $\phi$ green edges. Let $F$ denote the set of edges visited exactly once by $\langle W(t)\rangle$. Arbitrarily assigning some order to $L$, let $\left(p_{1}, q_{1}\right)$ denote the first link, and choose some $\left(x_{\langle 2 i\rangle}, x_{\langle 2 i+1\rangle}\right) \in F$ uniformly at random. We reroute the edge $\left(x_{\langle 2 i\rangle}, x_{\langle 2 i+1\rangle}\right)$ through $\left(p_{1}, q_{1}\right)$, forming

$$
W_{1}=\left(x_{\langle 0\rangle}, \ldots, x_{\langle 2 i\rangle}, p_{1}, q_{1}, x_{\langle 2 i+1\rangle}, \ldots, x_{\langle k\rangle}\right) .
$$

Form $F_{1}$ from $F$ by replacing $\left(x_{\langle 2 i\rangle}, x_{\langle 2 i+1\rangle}\right)$ by $\left(x_{\langle 2 i\rangle}, p_{1}\right)$ and $\left(q_{1}, x_{\langle 2 i+1\rangle}\right)$. We repeat the above using $F_{1}$ with the next $\left(p_{2}, q_{2}\right) \in L$, forming $W_{2}, W_{3}, \ldots$, until all links have been placed in the walk. The final walk is $W(t)$. We refer to this as sprinkling the links into the green edges of the contracted walk. Note that this can be viewed as a Pólya urn process; the urns are the edges of $F$, and the number of balls in urn $\left(x_{\langle 2 i\rangle}, x_{\langle 2 i+1\rangle}\right) \in F$ is the length of the path between the two points in $W(t)$. Indeed, this value is initially 1 for each member of $F$, and if a member of $F$ is picked then it is replaced by two members, and so on.

\subsection{Set structure}

Lemma 12. Suppose $[W(t)]$ is an equivalence class with $r n-2 t+1$ free configuration points, where $r n-2 t \rightarrow \infty$ and $r n-2 t=o(n)$, and suppose $\Phi(t) \geqslant n \delta^{1 / 2}, X_{1}^{g}(t)=r n \delta(1-$ $o(1)$ ) and $Z(t)=O\left(n \delta^{3 / 2}\right)$. Let $\omega$ tend to infinity arbitrarily slowly. If $W(t)$ is chosen uniformly at random from $[W(t)]$, then with high probability, the set $\bar{X}(t)$ associated with $W(t)$ is a root set of order $\omega$. 
Proof. Say that $v \in X_{1}^{g}$ is bad if its distance to the rest of $\bar{X}$ is at most $\omega$, or if it lies on a cycle of length at most $\omega$. Otherwise it is good. As $X_{1}^{g}$ makes up almost all of $\bar{X}=X_{1}^{g} \cup Z$, to show that $\bar{X}$ is a root set of order $\omega$ it is enough to show that the number of bad vertices in $X_{1}^{g}$ is at most $X_{1}^{g} / \omega^{3}$.

By Lemma 3, at most $\omega r^{\omega}=o\left(X_{1}^{g}(t)\right)$ vertices lie on cycles of length at most $\omega$. Let $\ell_{1}, \ell_{2} \in L$ be links. As they are sprinkled onto the $\phi=\Omega\left(n \delta^{1 / 2}\right)$ green edges of $[W(t)]$, the probability that they are placed within distance $\omega$ of each other is $O\left(r^{\omega} / \phi(t)\right)$. Indeed, any green edge in $[W(t)]$ is within graph distance at most $\omega$ of at most $r^{\omega}$ other green edges. The expected number of pairs $\ell_{1}, \ell_{2}$ within distance $\omega$ of each other is bounded by

$$
\sum_{\ell_{1} \neq \ell_{2}} O\left(\frac{r^{\omega}}{\phi(t)}\right)=O\left(\frac{L(t)^{2} r^{\omega}}{\phi(t)}\right)=O\left(\frac{n^{2} \delta^{2} r^{\omega}}{n \delta^{1 / 2}}\right)=o(n \delta) .
$$

As $X_{1}^{g}=\Omega(n \delta)$, choosing $\omega$ small enough this shows that all but $\bar{X}(t) / \omega^{3}$ of the links are at distance at least $\omega$ from other links. We conclude that $\bar{X}(t)$ is a root set of order $\omega$.

\section{Calculating the cover time}

Recall the definitions (10) of $\delta_{i}$ and $t_{i}, 0 \leqslant i \leqslant 4$. We begin by showing that the time taken to find the first $t_{1}$ edges contributes insignificantly to the cover time.

\section{Lemma 13.}

$$
\mathbb{E}\left(C\left(t_{1}\right)\right)=o(n \log n) .
$$

This is proved in Section 6.1. We then move on to estimating the expected cover time increment for larger $t$.

Lemma 14. For $t_{1} \leqslant t \leqslant t_{4}$ and any $\varepsilon>0$,

$$
\mathbb{E}(C(t+1)-C(t))=\left(\frac{r}{r-2} \pm \varepsilon\right) \frac{n}{r n-2 t} .
$$

Lemma 14 is proved in Section 6.2. The time to discover the final $O(\log n)$ edges can be bounded using (2). The increment $C(t+1)-C(t)$ is the number of steps taken by a simple random walk to reach a set of configuration points of size $r n-2 t+1$, which corresponds to a vertex $S$ of size at least $(r n-2 t+1) / r$. By $(2)$, we then have

$$
\mathbb{E}(C(t+1)-C(t))=O\left(\log n+\frac{n}{r n-2 t}\right),
$$

which implies

$$
\mathbb{E}\left(C\left(\frac{r n}{2}\right)-C\left(t_{4}\right)\right) \leqslant \sum_{s=t_{4}}^{r n / 2-1} O\left(\log n+\frac{n}{r n-2 s}\right)=o(n \log n) .
$$


This shows that for $t \geqslant t_{1}$,

$$
\begin{aligned}
\mathbb{E}(C(t)) & =\mathbb{E}\left(C\left(t_{1}\right)\right)+\sum_{s=t_{1}}^{t-1} \mathbb{E}(C(s+1)-C(s)) \\
& =\frac{r \pm \varepsilon}{2(r-2)} n \log \left(\frac{r n}{r n-2 t+1}\right)+o(n \log n),
\end{aligned}
$$

proving the edge cover time statement of Theorem 2. The vertex cover time follows from a simple argument using Lemma 10 (iv): the walk is expected to cover all but $s$ vertices at time $\delta=((s+1) / n)^{2 / r}$, which accounts for the $r / 2$ factor separating the vertex and edge cover times. The details are in Section 6.3.

To prove Theorem 1 it remains to show that $\mathbb{E}_{G}(C(t)) \sim \mathbb{E}(C(t))$ for almost all fixed $r$-regular graphs $G$. This is done in Appendix A.

\subsection{Phase one: Proof of Lemma 13}

With $t_{1}$ as in (10), we show that $\mathbb{E}\left(C\left(t_{1}\right)\right)=o(n \log n)$. Suppose $W(t)=\left(x_{0}, x_{1}, \ldots, x_{k}\right)$ for some $t, k$. If $x_{k} \in \mathcal{P}(\bar{X}(t))$ then $x_{k+1}=\mu\left(x_{k}\right)$ is uniformly random inside $\mathcal{P}(\bar{X}(t)) \backslash$ $\left\{x_{k}\right\}$, and since $C(t+1)=C(t)+1$ in the event of $x_{k+1} \in \mathcal{P}\left(X_{2} \cup \cdots \cup X_{r}\right)$, we have

$$
\mathbb{E}(C(t+1)-C(t)) \leqslant 1+\mathbb{E}\left(C(t+1)-C(t) \mid x_{k+1} \in \mathcal{P}\left(X_{1}\right)\right) \operatorname{Pr}\left\{x_{k+1} \in \mathcal{P}\left(X_{1}\right)\right\},
$$

We use the following theorem of Ajtai, Komlós and Szemerédi [1] to bound the expected change when $x_{k+1} \in \mathcal{P}\left(X_{1}\right)$.

Theorem 15. Let $G=(V, E)$ be an r-regular graph on $n$ vertices, and suppose that each of the eigenvalues of the adjacency matrix with the exception of the first eigenvalue are at most $\lambda_{G}$ (in absolute value). Let $A$ be a set of cn vertices of $G$. Then for every $\ell$, the number of walks of length $\ell$ in $G$ which avoid $A$ does not exceed $(1-c) n\left((1-c) r+c \lambda_{G}\right)^{\ell}$.

The set $A$ of Theorem 15 is fixed. In our case we choose a point $x_{k+1}$ uniformly at random from $\mathcal{P}\left(X_{1}(t)\right)$, so we consider a simple random walk initiated at a uniformly random vertex $u \in X_{1}(t)$. The subsequent walk now begins at vertex $u$ and continues until it hits a vertex of $Y_{u}=\bar{X}(t) \backslash\{u\}$. Because the vertex $u$ is random, the set $Y_{u}$ differs for each possible exit vertex $u \in X_{1}(t)$. To apply Theorem 15, we split $X_{1}(t)$ into two disjoint sets $A, A^{\prime}$ of (almost) equal size. For $u \in A$, instead of considering the number of steps needed to hit $Y_{u}$, we can upper bound this by the number of steps needed to hit $B^{\prime}=A^{\prime} \cup X_{2} \cup \cdots \cup X_{r}$, and vice versa. Suppose without loss of generality that $u \in A$.

Let $S(\ell)$ be a simple random walk of length $\ell$ starting from a uniformly chosen vertex of $A$. Thus $S(\ell)$ could be any of $|A| r^{\ell}$ uniformly chosen random walks. Let $c=\left|B^{\prime}\right| / n$. The probability $p_{\ell}$ that a randomly chosen walk of length $\ell$ starting from $A$ has avoided $B^{\prime}$ is, by Theorem 15, at most

$$
p_{\ell} \leqslant \frac{1}{\left(\left|X_{1}(t)\right| / 2\right) r^{\ell}}(1-c) n\left(r(1-c)+c \lambda_{G}\right)^{\ell} \leqslant \frac{2(1-c) n}{\left|X_{1}(t)\right|}((1-c)+c \lambda)^{\ell},
$$


where $\lambda \leqslant .99$ (see Lemma 3 ) is the absolute value of the second largest eigenvalue of the transition matrix of $S$. Thus

$$
\mathbb{E}_{A}(H(C)) \leqslant \sum_{\ell \geqslant 1} p_{\ell} \leqslant \frac{2(1-c) n}{\left|X_{1}(t)\right|} \frac{1}{c(1-\lambda)} .
$$

So,

$$
\mathbb{E}\left(C(t+1)-C(t) \mid x_{2 k} \in \mathcal{P}\left(X_{1}(t)\right)\right)=O\left(\frac{\left(n-\left|B^{\prime}\right|\right) n}{\left|X_{1}\right|\left|B^{\prime}\right|}\right) .
$$

Now, for any $t$ we have $r^{-1}(r n-2 t) \leqslant\left|B^{\prime}\right| \leqslant r n-2 t$, and the probability that $x_{k+1} \in X_{1}$ is $\left|X_{1}\right| /(r n-2 t+1)$. Summing over $0 \leqslant t \leqslant t_{1}$, (13) gives

$$
\mathbb{E}\left(C\left(t_{1}\right)\right)=\sum_{t=0}^{t_{1}}\left(1+O\left(\frac{n^{2}}{(r n-2 t)^{2}}\right)\right)=o(n \log n) .
$$

\subsection{Phase two: Proof of Lemma 14}

Lemma 16. Let $\varepsilon>0$. For $t_{1} \leqslant t \leqslant t_{4}$,

$$
\mathbb{E}(C(t+1)-C(t))=\left(\frac{r}{r-2} \pm \varepsilon\right) \frac{n}{r n-2 t} .
$$

Proof. The proof of Lemma 14 is based on the following calculation. Define events

$$
\begin{aligned}
& \mathcal{A}(t)=\left\{\left|X_{1}^{g}(t)-(r n-2 t)\right| \leqslant \frac{r n-2 t}{\omega}\right\}, \\
& \mathcal{B}(t)=\{\bar{X}(t) \text { is a root set of order } \omega\},
\end{aligned}
$$

and set $\mathcal{E}(t)=\mathcal{A}(t) \cap \mathcal{B}(t)$. Then for any $\varepsilon>0$, Lemma 7 shows that $\mathbb{E}(C(t+1)-C(t))$ can be calculated as

$$
\left[\left(\frac{r}{r-2} \pm \varepsilon\right) \frac{n}{r n-2 t}+O(\log n)\right] \operatorname{Pr}\{\mathcal{E}(t)\}+O\left(\frac{n}{r n-2 t} \operatorname{Pr}\{\overline{\mathcal{E}(t)}\}\right)
$$

Indeed, suppose $\mathcal{E}(t)$ holds. As $X_{1}(t)$ contains almost all unvisited configuration points, edge $t$ is attached to some $v \in X_{1}(t)$ w.h.p., and a simple random walk commences at $v$, ending once it hits $\bar{X} \backslash\{v\}$. As $\mathcal{A}(t)$ holds we have $|\bar{X}| \sim r n-2 t$. If $\mathcal{E}(t)$ does not hold, then we use the bound (1), stating that the hitting time is $O(n /|\bar{X}|)=O(n /(r n-2 t))$ (as $|\bar{X}| \geqslant(r n-2 t) / r$ ) as long as the graph has a positive eigenvalue gap. We refer to the discussion in Section 3 justifying our assumption that the second largest eigenvalue stays at most 0.99 throughout the process.

Lemmas 10 and 12 show that indeed, $\operatorname{Pr}\{\mathcal{E}(t)\}=1-o(1)$ for $t_{1} \leqslant t \leqslant t_{3}$. It remains to show that $\operatorname{Pr}\{\mathcal{E}(t)\}=1-o(1)$ for $t_{3} \leqslant t \leqslant t_{4}$. Fix $t>t_{3}$. As $Z\left(t_{3}\right)=O\left(n \delta_{3}^{3 / 2}\right)=o(1)$, 
we have $Z(t) \subseteq Z\left(t_{3}\right)=\emptyset$ w.h.p. by Markov's inequality. Note that this implies $X_{1}^{g}(t) \subseteq$ $X_{1}^{g}\left(t_{3}\right)$ and $\left|X_{1}^{g}(t)\right|=r n-2 t$. We also have

$$
\frac{L\left(t_{3}\right)^{2}}{\Phi\left(t_{3}\right)}=O\left(\frac{n^{2} \delta_{3}^{2}}{n \delta_{3}^{1 / 2}}\right)=O\left(n\left(n^{-3 / 4}\right)^{3 / 2}\right)=o(1) .
$$

Thus, repeating the calculation (12) of Lemma 12, we have that no two vertices of $X_{1}^{g}\left(t_{3}\right)$ are placed within distance $\omega$ of each other. As $X_{1}^{g}(t) \subseteq X_{1}^{g}\left(t_{3}\right)$, the same must be true of $X_{1}^{g}(t)$. Thus the only vertices of $X_{1}^{g}(t)$ which violate the root set constraints are those placed on the $\omega r^{\omega}$ short cycles of $G_{r}$, and choosing $\omega$ small enough we have $\omega r^{\omega} \leqslant(r n-2 t) / \omega^{3}$ for all $t_{3} \leqslant t \leqslant t_{4}$, so w.h.p. $\bar{X}(t)=X_{1}^{g}(t)$ is a root set of order $\omega$.

\subsection{The vertex cover time}

Using Lemma 10 (iv) we can move between partial edge cover time $C(t)=C_{E}(t)$ and partial vertex cover time $C_{V}(t)$. The following lemma is almost exactly as the one in [5] for the case $r=3$.

Lemma 17. Let $\left(1-\log ^{-r} n\right) n \leqslant s \leqslant n$. Then

$$
\mathbb{E}\left(C_{V}(s)\right)=\left(\frac{1}{r-2} \pm \varepsilon\right) n \log \left(\frac{n}{n-s+1}\right) .
$$

Proof. The set of undiscovered vertices at time $t$ is $X_{r}(t)$. We write $C_{V}(s)=C\left(\tau_{s}\right)$, where

$$
\tau_{s}=\min \left\{t:\left|X_{r}(t)\right|=n-s\right\} .
$$

Suppose $n-s$ tends to infinity with $n$. Let $\delta_{s}=(1-(s-1) / n)^{2 / r}$, and let $\omega$ tend to infinity arbitrarily slowly with $n$. Define $\tau_{s}^{-}=\left(1-\delta_{s} \omega\right) \frac{r n}{2}$ and $\tau_{s}^{+}=\left(1-\delta_{s} \omega^{-1}\right) \frac{r n}{2}$. By Lemma 10 (iv), w.h.p.,

$$
X_{r}\left(\tau_{s}^{-}\right) \approx n\left(\delta_{s} \omega\right)^{r / 2}=n\left(1-\frac{s-1}{n}\right) \omega^{r / 2} \gg n-s,
$$

and similarly, w.h.p. $X_{r}\left(\tau_{s}^{+}\right) \ll n-s$. In particular, w.h.p. $\tau_{s}^{-}<\tau_{s}<\tau_{s}^{+}$. We have

$$
\begin{aligned}
\mathbb{E}\left(C_{V}(s)\right)=\mathbb{E}\left(C\left(\tau_{s}\right)\right) & =\mathbb{E}\left(C\left(\tau_{s}^{+}\right)\right)+\mathbb{E}\left(C\left(\tau_{s}\right)-C\left(\tau_{s}^{+}\right)\right) \\
& \leqslant \mathbb{E}\left(C\left(\tau_{s}^{+}\right)\right)+\mathbb{E}\left(\left(C\left(\tau_{s}\right)-C\left(\tau_{s}^{+}\right)\right) \mathbf{1}_{\tau_{s}>\tau_{s}^{+}}\right) \\
& \leqslant \mathbb{E}\left(C\left(\tau_{s}^{+}\right)\right)+\mathbb{E}\left(C(T)-C\left(\tau_{s}^{+}\right) \mid \tau_{s}>\tau_{s}^{+}\right) \operatorname{Pr}\left\{\tau_{s}>\tau_{s}^{+}\right\},
\end{aligned}
$$

where $T=\left(1-\frac{n-s}{n}\right) \frac{r n}{2}$ is an upper bound for $\tau_{s}$. Indeed, at time $T$ there are $r n-2 T<$ $r(n-s)$ unvisited configuration points, so $\tau_{s}<T$. Conditional on the event $\left\{\tau_{s}>\tau_{s}^{+}\right\}$, for $t \geqslant \tau_{s}^{+}$we can apply (2) to obtain

$$
\mathbb{E}\left(C(t+1)-C(t) \mid \tau_{s}>\tau_{s}^{+}\right)=O\left(\log n+\frac{n}{r n-2 t}\right) .
$$


We then have

$$
\begin{aligned}
\mathbb{E}\left(C(T)-C\left(\tau_{s}^{+}\right) \mid \tau_{s}>\tau_{s}^{+}\right) & =O\left(\left(T-\tau_{s}^{+}\right) \log n+n \log \left(\frac{r n-2 T}{r n-2 \tau_{s}^{+}}\right)\right) \\
& =O\left(n \log \left(\frac{n}{n-s}\right)\right)
\end{aligned}
$$

We calculate $\mathbb{E}\left(C\left(\tau_{s}^{+}\right)\right)$using Theorem 2 to obtain

$$
\begin{aligned}
\mathbb{E}\left(C_{V}(s)\right) & \leqslant \frac{r+\varepsilon}{2(r-2)} n \log \left(\frac{1}{\left(\delta_{s} \omega^{-1}\right)^{r / 2}}\right)+o\left(n \log \left(\frac{n}{n-s}\right)\right) \\
& =\frac{1+\varepsilon}{r-2} n \log \left(\frac{n}{n-s}\right) .
\end{aligned}
$$

For a lower bound, we use

$$
\mathbb{E}\left(C_{V}(s)\right) \geqslant \mathbb{E}\left(C\left(\tau_{s}^{-}\right)\right)-\mathbb{E}\left(C\left(\tau_{s}^{-}\right)-C\left(\tau_{s}\right) \mid \tau_{s}<\tau_{s}^{-}\right) \operatorname{Pr}\left\{\tau_{s}<\tau_{s}^{-}\right\},
$$

and using the same argument as above it is enough to note that for $t \leqslant \tau_{s}^{-}$,

$$
\mathbb{E}\left(C(t+1)-C(t) \mid \tau_{s} \leqslant t\right)=O\left(\log n+\frac{n}{r n-2 t}\right) .
$$

\section{Set sizes}

Recall the definition

$$
Z(t)=X_{1}^{b}(t) \cup \bigcup_{i=2}^{r} X_{i}(t),
$$

where $X_{i}$ denotes the set of vertices incident to $i$ unvisited edges, and $X_{1}^{b}$ is the set of vertices in $X_{1}$ which are incident to at least one edge which has been visited more than once.

Lemma 18. There exists a constant $B>0$ such that for $t \geqslant t_{0}$ and $0<\theta=o(1)$,

$$
\mathbb{E}\left(e^{\theta Z(t)}\right) \leqslant \exp \left\{\theta B n \delta^{3 / 2}\right\} .
$$

Proof. We show that there exists a $B>0$ such that for any $m \geqslant 1$,

$$
\operatorname{Pr}\{[m] \subseteq Z(t)\} \leqslant(B \delta)^{3 m / 2},
$$

beginning with $m=1$ before the general statement. Note that any vertex $v \in Z(t)$ has been visited some number $0 \leqslant k<r$ of times. To be precise, there are $k$ indices $i_{1}, \ldots, i_{k}$ such that $x_{2 i_{j}+1} \in \mathcal{P}(v)$. Let $\ell_{j} \in\{1,2\}$ denote the number of new edges incident to $v$ in the segment $\left(x_{2 i_{j}-1}, x_{2 i_{j}}, x_{2 i_{j}+1}, x_{2 i_{j}+2}\right)$; the edge $\left(x_{2 i_{j}+1}, x_{2 i_{j}+2}\right)$ must be new by design of the random walk, while $\left(x_{2 i_{j}-1}, x_{2 i_{j}}\right)$ may or may not appear earlier in the walk. Let 
$\mathcal{L}=\mathcal{L}(r)$ denote the set of vectors $\left(\ell_{1}, \ell_{2}, \ldots, \ell_{k}\right)$ with $\ell_{i} \in\{1,2\}$ such that $\sum \ell_{i} \leqslant r-1$, including in $\mathcal{L}$ the empty vector $\emptyset$ (for unvisited vertices), excluding the vector $(2,2, \ldots, 2)$ consisting of $(r-1) / 2$ copies of 2 (for green vertices). We partition

$$
Z(t)=\bigcup_{\ell \in \mathcal{L}} Z_{\ell}(t)
$$

where $v \in Z_{\ell}(t)$ for $\ell=\left(\ell_{1}, \ldots, \ell_{k}\right)$ if and only if there exists a sequence $0<s_{1}<$ $s_{2}<\cdots<s_{k} \leqslant t$ such that $v$ moves from $X_{r-\ell_{1}-\cdots-\ell_{j-1}}$ to $X_{r-\ell_{1}-\cdots-\ell_{j}}$ at time $s_{j}$ for $j=1, \ldots, k$, and is in $X_{r-\ell_{1}-\cdots-\ell_{k}}$ at time $t$. If $v \in X_{i}$ at time $s$, the probability that $v$ is chosen by random assignment is $i /(r n-2 s)$, while Lemma 8 shows that the probability that $v$ is at the end of a blue walk is $O(1 /(r n-2 s))$. In either case, the probability that $v$ moves from one set to another is at most $B /(r n-2 s)$ for some $B>0$. For a fixed $\ell=\left(\ell_{1}, \ldots, \ell_{k}\right) \in \mathcal{L}$, with $s_{0}=1$,

$$
\begin{aligned}
\operatorname{Pr}\left\{1 \in Z_{\ell}(t)\right\} \leqslant & \sum_{s_{1}<\cdots<s_{k}} \prod_{j=1}^{k}\left[\prod_{s=s_{j-1}+1}^{s_{j}-1}\left(1-\frac{r-\left(\ell_{1}+\cdots+\ell_{j-1}\right)}{r n-2 s}\right) \frac{B}{r n-2 s_{j}}\right] \\
& \times \prod_{s=s_{k}+1}^{t}\left(1-\frac{r-\left(\ell_{1}+\cdots+\ell_{k}\right)}{r n-2 s}\right) .
\end{aligned}
$$

For $b \geqslant 1$ we use the bound

$$
\begin{aligned}
\prod_{s=t_{0}}^{t}\left(1-\frac{b}{r n-2 s}\right) & \leqslant \exp \left\{-\frac{b}{2} \sum_{s=t_{0}}^{t} \frac{1}{\frac{r n}{2}-s}\right\} \\
& \leqslant \exp \left\{-\frac{b}{2} \int_{t_{0}}^{t} \frac{d x}{\frac{r n}{2}-x}\right\} \\
& =\left(\frac{r n-2 t}{r n-2 t_{0}}\right)^{b / 2} .
\end{aligned}
$$

Combining (15) and (16), the probability that $1 \in Z_{\ell}(t)$ is bounded above by

$$
\sum_{s_{1}<\cdots<s_{k}}\left[\prod_{j=1}^{k} \frac{B}{r n-2 s_{j}}\left(\frac{r n-2 s_{j}}{r n-2 s_{j-1}}\right)^{\left(r-\left(\ell_{1}+\cdots+\ell_{j-1}\right)\right) / 2}\right]\left(\frac{r n-2 t}{r n-2 s_{k}}\right)^{\left(r-\left(\ell_{1}+\cdots+\ell_{k}\right)\right) / 2} .
$$

Collecting powers of $r n-2 s_{j}$ for $j=1, \ldots, k$, we have

$$
\operatorname{Pr}\left\{1 \in Z_{\ell}(t)\right\} \leqslant B^{k} \frac{(r n-2 t)^{\left(r-\left(\ell_{1}+\cdots+\ell_{k}\right)\right) / 2}}{(r n)^{r / 2}} \sum_{s_{1}<\cdots<s_{k}} \prod_{j=1}^{k}\left(r n-2 s_{j}\right)^{\ell_{j} / 2-1} .
$$


Let $N$ denote the number of indices $j \in\{1, \ldots, k\}$ with $\ell_{j}=1$. Then

$$
\begin{aligned}
\sum_{s_{1}<\cdots<s_{k}} \prod_{j=1}^{k}\left(r n-2 s_{j}\right)^{\ell_{j} / 2-1} & \leqslant \prod_{j=1}^{k}\left(\sum_{s=0}^{t}(r n-2 s)^{\ell_{j} / 2-1}\right) \\
& \leqslant(t+1)^{k-N} \prod_{j: \ell_{j}=1}\left(\sum_{s=0}^{t}(r n-2 s)^{-1 / 2}\right) \\
& \leqslant(r n)^{k-N}(r n-2 t)^{N / 2}
\end{aligned}
$$

which implies that

$$
\operatorname{Pr}\left\{1 \in Z_{\ell}(t)\right\} \leqslant \frac{B^{k}}{r^{r / 2}}(r n-2 t)^{\left(r+N-\left(\ell_{1}+\cdots+\ell_{k}\right)\right) / 2}(r n)^{k-N-r / 2} .
$$

As $\ell_{1}+\cdots+\ell_{k}=2 k-N$, we have $\left(r+N-\left(\ell_{1}+\cdots+\ell_{k}\right)\right) / 2=r / 2-k+N$. So

$$
\operatorname{Pr}\left\{1 \in Z_{\ell}(t)\right\} \leqslant \frac{B^{k}}{r^{r-k+N}} \delta^{r / 2-k+N} .
$$

We now argue that $r / 2-k+N \geqslant 3 / 2$, or equivalently $2(k-N) \leqslant r-3$, for all $\ell \in \mathcal{L}$. Firstly, if $\ell_{1}+\cdots+\ell_{k} \leqslant r-3$ then we have $2(k-N) \leqslant 2 k-N=\ell_{1}+\cdots+\ell_{k} \leqslant r-3$. Secondly, if $\ell_{1}+\cdots+\ell_{k}=r-2$ then as $r-2$ is odd we have $N \geqslant 1$, so $2(k-N) \leqslant$ $2 k-N-1 \leqslant r-3$. Finally, if $\ell_{1}+\cdots+\ell_{k}=r-1$ then $($ as $(2,2, \ldots, 2) \notin \mathcal{L})$ we have $N \geqslant 2$, so $2(k-N) \leqslant 2 k-N-2 \leqslant r-3$.

As $|\mathcal{L}(r)|$ is a function of $r$ only, and therefore constant with respect to $n$, it follows that

$$
\operatorname{Pr}\{1 \in Z(t)\}=\sum_{\ell \in \mathcal{L}(r)} \operatorname{Pr}\left\{1 \in Z_{\ell}(t)\right\}=O\left(\delta^{3 / 2}\right) .
$$

We turn to bounding the probability that $[m] \subseteq Z(t)$. We fix $\ell^{(1)}, \ldots, \ell^{(m)} \in \mathcal{L}$ and bound the probability that $i \in Z_{\ell^{(i)}}(t)$ for $i=1, \ldots, m$. Let $k(i)=\operatorname{dim} \ell^{(i)}$ denote the number of components of $\ell^{(i)}$. Then, summing over all choices $s_{j}^{(i)}$ for $1 \leqslant i \leqslant m$ and $1 \leqslant j \leqslant k(i)$

$$
\begin{aligned}
& \operatorname{Pr}\left\{i \in Z_{\ell^{(i)}}(t), i=1, \ldots, m\right\} \\
& \leqslant \sum_{s_{j}^{(i)}} \prod_{i=1}^{m} B^{k(i)} \frac{(r n-2 t)^{\left(r-\sum_{j} \ell_{j}^{(i)}\right) / 2}}{(r n)^{r / 2}} \prod_{j=1}^{k(i)}\left(r n-2 s_{j}^{(i)}\right)^{\ell_{j}^{(i)} / 2-1} \\
& \leqslant \prod_{i=1}^{m}\left[B^{k(i)} \frac{(r n-2 t)^{\left(r-\sum_{j} \ell_{j}^{(i)}\right) / 2}}{(r n)^{r / 2}} \prod_{j=1}^{k(i)}\left(\sum_{s=0}^{t}(r n-2 s)^{\ell_{j}^{(i)} / 2-1}\right)\right] \\
& \leqslant B^{\sum k(i)} \delta^{3 m / 2}=O\left(\left(B^{r} \delta\right)^{3 m / 2}\right) .
\end{aligned}
$$

Summing over all $O(m)$ choices of $\ell^{(i)}, i=1, \ldots, m$, we have

$$
\operatorname{Pr}\{[m] \subseteq Z(t)\}=O\left(m\left(B^{r} \delta\right)^{3 m / 2}\right) \leqslant(C \delta)^{3 m / 2}
$$


for some constant $C>0$. By symmetry the same bound holds for any vertex set of size $m$. It follows that for any $m$, writing $(n)_{m}=n(n-1) \ldots(n-m+1)$,

$$
\mathbb{E}\left((Z(t))_{m}\right) \leqslant(n)_{m} \times(C \delta)^{3 m / 2} \leqslant\left(C n \delta^{3 / 2}\right)^{m} .
$$

For $s>1$ we apply the binomial theorem to obtain

$$
\mathbb{E}\left(s^{Z(t)}\right)=\mathbb{E}\left((1+(s-1))^{Z(t)}\right)=\sum_{m \geqslant 0} \frac{\mathbb{E}\left((Z(t))_{m}\right)(s-1)^{m}}{m !} .
$$

We set $s=e^{\theta} \leqslant 1+2 \theta(\operatorname{as} \theta=o(1))$ to obtain

$$
\mathbb{E}\left(e^{\theta Z(t)}\right) \leqslant \sum_{m \geqslant 0} \frac{\left(C n \delta^{3 / 2}\right)^{m}(2 \theta)^{m}}{m !} \leqslant \exp \left\{\theta D n \delta^{3 / 2}\right\}
$$

for $D \geqslant 2 C$.

Corollary 19. For $t=(1-\delta) \frac{r n}{2}$ with $\delta=o(1)$, and $0<\theta=o(1)$,

$$
\mathbb{E}\left(e^{-\theta X_{1}^{g}(t)}\right)=\exp \{-\theta r n \delta(1-o(1))\}
$$

Proof. The number of free configuration points at time $t$ is $r n-2 t$, so

$$
r n-2 t=\sum_{i=1}^{r} i X_{i}(t) \leqslant X_{1}^{g}(t)+r Z(t) .
$$

By Lemma 18 we have

$$
\mathbb{E}\left(e^{-\theta X_{1}^{g}(t)}\right) \leqslant e^{-\theta(r n-2 t)} \mathbb{E}\left(e^{r \theta Z(t)}\right)=\exp \{-\theta r n \delta(1-o(1))\} .
$$

The technique used to prove Lemma 18 can be strengthened to obtain concentration for the number of unvisited vertices $X_{r}(t)$. This is the topic of the next lemma.

Lemma 20. For $\theta>0$,

$$
\mathbb{E}\left(e^{\theta X_{r}(t)}\right) \leqslant \exp \left\{2 \theta n \delta^{r / 2}\right\} .
$$

Furthermore, if $t=(1-\delta) \frac{r n}{2}$ with $\delta=o(1)$ and $n \delta^{r / 2} \rightarrow \infty$, then for any $\omega$ tending to infinity arbitrarily slowly,

$$
\operatorname{Pr}\left\{\left|X_{r}(t)-n \delta^{r / 2}\right|>\frac{n \delta^{r / 2}}{\omega^{1 / 2}}\right\} \leqslant \frac{1}{\omega} .
$$

Finally, if $n \delta^{r / 2}=o(1)$ then $X_{r}(t)=0$ w.h.p. 
Proof. Suppose a biased random walk is initiated at a vertex chosen uniformly at random. The probability that no vertex in $[m]$ is not incident to any of the first $t$ distinct edges visited by the walk is bounded by

$$
\operatorname{Pr}\left\{[m] \subseteq X_{r}(t)\right\}=\left(1-\frac{m}{n}\right) \prod_{s=0}^{t}\left(1-\frac{r m}{r n-2 s-1}\right) \leqslant \delta^{r m / 2} .
$$

The factor $1-m / n$ is the probability that the initial vertex is in $[m]$, and the bound otherwise is exactly as in (16). The inequality (17) follows from the arguments used in the proof of Lemma 18 .

For $m=1$ we need the converse inequality to (16). From $1-x \geqslant e^{-x /(1-x)}$, valid for $x \neq 1$, we have

$$
\begin{aligned}
\prod_{s=0}^{t}\left(1-\frac{r}{r n-2 s}\right) & \geqslant \exp \left\{-\sum_{s=0}^{t} \frac{r}{r n-2 s-r}\right\} \\
& =\left(\frac{r n-2 t}{r n}\right)^{r / 2}(1-o(1)) .
\end{aligned}
$$

Together with (16) this shows that $\mathbb{E}\left(X_{r}(t)\right)=n \delta^{r / 2}(1-o(1))$. From (18) we have $\mathbb{E}\left(\left(X_{r}(t)\right)_{2}\right) \leqslant n(n-1) \delta^{r}$. We conclude that the leading terms of $\mathbb{E}\left(\left(X_{r}(t)\right)_{2}\right)$ and $\mathbb{E}\left(X_{r}(t)\right)^{2}$ agree, and

$$
\operatorname{Var}\left(X_{r}(t)\right)=\mathbb{E}\left(\left(X_{r}(t)\right)_{2}\right)+\mathbb{E}\left(X_{r}(t)\right)-\mathbb{E}\left(X_{r}(t)\right)^{2}=o\left(\mathbb{E}\left(X_{r}(t)\right)^{2}\right) .
$$

We apply Chebyshev's inequality with some $\omega$ tending to infinity sufficiently slowly:

$$
\operatorname{Pr}\left\{\left|X_{r}(t)-\mathbb{E}\left(X_{r}(t)\right)\right| \geqslant \frac{\mathbb{E}\left(X_{r}(t)\right)}{\omega^{1 / 2}}\right\} \leqslant \frac{\operatorname{Var}\left(X_{r}(t)\right) \omega}{\mathbb{E}\left(X_{r}(t)\right)^{2}}=o(1) .
$$

Finally, if $n \delta^{r / 2}=o(1)$ then $\mathbb{E}\left(X_{r}(t)\right) \leqslant n \delta^{r / 2}=o(1)$ and Markov's inequality shows that $\left|X_{r}(t)\right|=0$ w.h.p.

Lemma 20 relates the number of unvisited edges to the number of unvisited vertices: we expect $\left|X_{r}(t)\right|=n-s$ to occur when $t \sim\left(1-\frac{s}{n}\right)^{2 / r}$. This heuristically explains why $C_{E}^{b}\left(G_{r}\right) \sim \frac{r}{2} C_{V}^{b}\left(G_{r}\right)$. See Section 6.3 for details.

\section{The green edges}

Let $\Phi(t)$ denote the number of green edges in $W(t)$.

Lemma 21. Let $0<\varepsilon<r-2$ and define

$$
\delta_{\varepsilon}=\left(\frac{\log ^{4} n}{n}\right)^{\frac{r-1}{r+\varepsilon}}, \quad t_{\varepsilon}=\left(1-\delta_{\varepsilon}\right) \frac{r n}{2} .
$$

Then with high probability, $\Phi(t) \geqslant n \delta^{\frac{1+\varepsilon}{r-1}}$ for all $t_{1} \leqslant t \leqslant t_{\varepsilon}$. 
With $\varepsilon>0$ small enough so that $(r-1) /(r+\varepsilon)>3 / 4$ and $(1+\varepsilon) /(r-1)<1 / 2$, Lemma 21 implies statement (ii) of Lemma 10: if $t_{1} \leqslant t \leqslant t_{3}$ then w.h.p., $\Phi(t) \geqslant n \delta^{1 / 2}$.

Proof of Lemma 21. Firstly, let us see how $\Phi(t)$ changes with time. Fix $\varepsilon_{1}>0$ such that

$$
\frac{1}{\left(1-\varepsilon_{1}\right)(r-1)}<\frac{1+\varepsilon}{r-1}
$$

let

$$
\mathcal{X}(t)=\left\{X_{1}^{g}(t) \geqslant\left(1-\varepsilon_{1}\right)(r n-2 t)\right\}
$$

and let $\mathbf{1}_{t}$ denote the indicator variable for $\mathcal{X}(t)$. We note that with $\lambda=1 / \log n$, by Corollary 19

$$
\operatorname{Pr}\{\overline{\mathcal{X}(t)}\} \leqslant \frac{\mathbb{E}\left(e^{-\lambda X_{1}^{g}(t)}\right)}{e^{-\lambda\left(1-\varepsilon_{1}\right)(r n-2 t)}} \leqslant \exp \left\{-\frac{\varepsilon_{1} n \delta_{\varepsilon}}{\log n}\right\}=: \eta
$$

for any $t \leqslant t_{\varepsilon}$.

Claim 22. For $0<\theta \leqslant \delta_{\varepsilon} \log ^{-2} n, \varepsilon_{1}>0$ and $t_{0} \leqslant t \leqslant t_{\varepsilon}$,

$$
\mathbb{E}\left(e^{-\theta(\Phi(t+1)-\Phi(t))} \mathbf{1}_{t} \mid[W(t)]\right) \leqslant \exp \left\{\frac{2 \theta \Phi(t)}{\left(1-\varepsilon_{1}\right)(r-1)(r n-2 t)}(1+O(\gamma))\right\} \mathbf{1}_{t},
$$

with $\gamma=o\left(\log ^{-1} n\right)$.

Proof. Condition on a $[W(t)]$ such that $X_{1}^{g}(t) \geqslant\left(1-\varepsilon_{1}\right)(r n-2 t)$, recalling that the set $X_{1}^{g}(t)$ is the same for all walks in $[W(t)]$, as mentioned in Section 5.2. If the next edge is added without entering a blue walk, then $\Phi(t+1)=\Phi(t)+1$. So,

$$
\operatorname{Pr}\{\Phi(t+1)-\Phi(t)=1 \mid[W(t)]\}=1-\frac{X_{1}(t)}{r n-2 t} .
$$

Suppose the new edge chooses a vertex of $X_{1}(t)$, thus entering a blue walk. We may view this as a walk on $[W(t)]$, and any time a green edge is traversed, we ask if the green edge in $[W(t)]$ contains a green link in $W(t)$, in which case the blue walk ends. If not, the green edge turns blue and $\Phi$ decreases by one.

There are $L(t)=\frac{r-1}{2} X_{1}^{g}(t)$ green links, distributed into the $\Phi(t)$ green edges by a Pólya urn process as discussed in Section 5. Suppose $e_{1}, e_{2}, \ldots, e_{\ell}$ are green edges in $[W(t)]$, and let $K_{1}, K_{2}, \ldots, K_{\ell}$ be the lengths of the corresponding paths in $W(t)$, corresponding to the first $\ell$ entries of a vector $\left(k_{1}, \ldots, k_{\phi(t)}\right)$ drawn uniformly at random from all vectors with $k_{i} \geqslant 1$ and $\sum_{i=1}^{\phi(t)} k_{i}=\Phi(t)$. The number of such vectors is $\left(\begin{array}{c}\Phi(t)-1 \\ \phi(t)-1\end{array}\right)$, and noting that $\Phi(t)=\phi(t)+L(t)$, the probability that none of the $\ell$ edges contain a green link is exactly

$$
\begin{aligned}
\operatorname{Pr}\left\{K_{i}=1 \text { for } i=1,2, \ldots, \ell\right\}=\frac{\left(\begin{array}{c}
\Phi(t)-\ell-1 \\
\phi(t)-\ell-1
\end{array}\right)}{\left(\begin{array}{c}
\Phi(t)-1 \\
\phi(t)-1
\end{array}\right)} & =\prod_{i=1}^{\ell} \frac{\phi(t)-i}{\Phi(t)-i} \\
& =\prod_{i=1}^{\ell}\left(1-\frac{L(t)}{\Phi(t)-i}\right) \leqslant\left(1-\frac{L(t)}{\Phi(t)}\right)^{\ell} .
\end{aligned}
$$


This shows that the number of green edges visited before discovering a green link can be bounded by a geometric random variable. If a green edge is visited without a discovery, that edge turns blue. Note that the blue walk also ends when a vertex of $X_{i}^{b}$ is found for some $i \geqslant 1$; we are upper bounding the number of green edges visited.

So in distribution,

$$
\Phi(t+1)-\Phi(t) \stackrel{d}{=} 1-B\left(\frac{X_{1}(t)}{r n-2 t}\right) R_{t}
$$

where $B(p)$ denotes a Bernoulli random variable taking value 1 with probability $p$, and $R_{t}$ is stochastically dominated above by a geometric random variable with success probability $L(t) / \Phi(t)$. The two random variables on the right-hand side are independent. So

$$
\mathbb{E}\left(e^{-\theta(\Phi(t+1)-\Phi(t))} \mid[W(t)]\right)=e^{-\theta}\left(1-\frac{X_{1}(t)}{r n-2 t}+\frac{X_{1}(t)}{r n-2 t} \mathbb{E}\left(e^{\theta R_{t}} \mid[W(t)]\right)\right)
$$

The map $x \mapsto e^{\theta x}$ is increasing for $\theta>0$, so we can couple $R_{t}$ to a geometric random variable $S_{t}$ with success probability $L(t) / \Phi(t)$ in such a way that

$$
\mathbb{E}\left(e^{\theta R_{t}} \mid[W(t)]\right) \leqslant \mathbb{E}\left(e^{\theta S_{t}} \mid[W(t)]\right) .
$$

As $S_{t}$ is geometrically distributed and $X_{1}^{g}(t) \geqslant(r n-2 t) / 2$ by conditioning on $\mathcal{X}(t)$,

$$
\mathbb{E}\left(e^{\theta S_{t}} \mid[W(t)]\right)=1+\theta \frac{\Phi(t)}{L(t)}-O\left(\frac{\theta^{2} \Phi(t)^{2}}{L(t)^{2}}\right)=1+\theta \frac{\Phi(t)}{L(t)}(1+O(\gamma)) .
$$

Conditioning on $X_{1}^{g}(t) \geqslant\left(1-\varepsilon_{1}\right)(r n-2 t)$ implies that $L(t)=\frac{r-1}{2} X_{1}^{g}(t)=\Omega(n \delta)$, so

$$
\gamma:=\theta \frac{\Phi(t)}{L(t)} \leqslant \delta_{\varepsilon} \log ^{-2} n \frac{n}{\Omega\left(n \delta_{\varepsilon}\right)}=o\left(\log ^{-1} n\right) .
$$

We also have $X_{1}^{b}(t) \leqslant r n-2 t-X_{1}^{g}(t) \leqslant \varepsilon_{1}(r n-2 t)$, so

$$
\frac{X_{1}(t)}{L(t)}=\frac{X_{1}^{g}(t)}{L(t)}+\frac{X_{1}^{b}(t)}{L(t)} \leqslant \frac{2}{r-1}+\frac{\varepsilon_{1}(r n-2 t)}{\left(1-\varepsilon_{1}\right) \frac{r-1}{2}(r n-2 t)}=\frac{2}{\left(1-\varepsilon_{1}\right)(r-1)} .
$$

So for $[W(t)] \in \mathcal{X}(t)$,

$$
\begin{aligned}
& \mathbb{E}\left(e^{-\theta(\Phi(t+1)-\Phi(t))} \mathbf{1}_{t} \mid[W(t)]\right) \\
& \leqslant e^{-\theta}\left(1-\frac{X_{1}(t)}{r n-2 t}+\frac{X_{1}(t)}{r n-2 t}\left(1+\theta \frac{\Phi(t)}{L(t)}(1+O(\gamma))\right)\right) \\
& \leqslant\left(1+\theta \frac{2 \Phi(t)}{\left(1-\varepsilon_{1}\right)(r-1)(r n-2 t)}(1+O(\gamma))\right) \\
& \leqslant \exp \left\{\frac{2 \theta \Phi(t)}{\left(1-\varepsilon_{1}\right)(r-1)(r n-2 t)}(1+O(\gamma))\right\} .
\end{aligned}
$$


Define for $0<\theta=o(1)$,

$$
f_{t}(\theta)=\mathbb{E}\left(e^{-\theta \Phi(t)} \mathbf{1}_{t}\right) .
$$

As $\Phi(t) \geqslant L(t)=\frac{r-1}{2} X_{1}^{g}(t)$ we have for $0<\theta=o(1)$, by Corollary 19 ,

$$
f_{t_{0}}(\theta) \leqslant \mathbb{E}\left(e^{-\theta \Phi\left(t_{0}\right)}\right) \leqslant \mathbb{E}\left(e^{-\theta \frac{r-1}{2} X_{1}^{g}(t)}\right)=\exp \left\{-\theta \frac{r-1}{2} r n \delta_{0}(1+o(1))\right\} .
$$

Claim 22 shows that for $t_{0} \leqslant t<t_{\varepsilon}$,

$$
\begin{aligned}
f_{t+1}(\theta) & =\mathbb{E}\left(e^{-\theta \Phi(t+1)} \mathbf{1}_{t}\right)+\mathbb{E}\left(e^{-\theta \Phi(t+1)}\left(\mathbf{1}_{t+1}-\mathbf{1}_{t}\right)\right) \\
& \leqslant \mathbb{E}\left(e^{-\theta \Phi(t)} \mathbb{E}\left(e^{-\theta(\Phi(t+1)-\Phi(t))} \mathbf{1}_{t} \mid[W(t)]\right)\right)+\mathbb{E}\left(1-\mathbf{1}_{t}\right) \\
& \leqslant \mathbb{E}\left(\exp \left\{-\theta \Phi(t)\left(1-\frac{2(1+O(\gamma))}{\left(1-\varepsilon_{1}\right)(r-1)(r n-2 t)}\right)\right\} \mathbf{1}_{t}\right)+\eta \\
& =f_{t}\left(\theta\left(1-\frac{2(1+O(\gamma))}{\left(1-\varepsilon_{1}\right)(r-1)(r n-2 t)}\right)\right)+\eta
\end{aligned}
$$

where $\eta=\exp \left\{-\varepsilon_{1} n \delta_{\varepsilon} / \log n\right\}$ is an upper bound for $\operatorname{Pr}\{\overline{\mathcal{X}(t)}\}$, as defined in (20). As $\gamma=o\left(\log ^{-1} n\right)$, repeating the calculations in (16) and (18), we have

$$
\prod_{s=t_{0}}^{t-1}\left(1-\frac{2(1+O(\gamma))}{\left(1-\varepsilon_{1}\right)(r-1)(r n-2 s)}\right) \sim\left(\frac{r n-2 t}{r n-2 t_{0}}\right)^{\frac{1}{\left(1-\varepsilon_{1}\right)(r-1)}} .
$$

It follows by induction and from (21) that

$$
\begin{aligned}
f_{t}(\theta) & \leqslant f_{t_{0}}\left(\theta \prod_{s=t_{0}}^{t-1}\left(1-\frac{2(1+O(\gamma))}{\left(1-\varepsilon_{1}\right)(r-1)(r n-2 s)}\right)\right)+\left(t-t_{0}\right) \eta \\
& \leqslant \exp \left\{-\theta r n \delta_{0}\left(\frac{\delta}{\delta_{0}}\right)^{\frac{1}{\left(1-\varepsilon_{1}\right)(r-1)}}\right\}+\left(t-t_{0}\right) \eta .
\end{aligned}
$$

Now, $\varepsilon_{1}$ was chosen in (19) to satisfy $1 /\left(1-\varepsilon_{1}\right)(r-1)<(1+\varepsilon) /(r-1)$. The $\delta_{0}=1 / \log \log n$ factors are insignificant compared to those involving $\delta \leqslant \log ^{-1 / 2} n$, and we have

$$
n \delta_{0}\left(\frac{\delta}{\delta_{0}}\right)^{\frac{1}{\left(1-\varepsilon_{1}\right)(r-1)}}>n \delta^{\frac{1+\varepsilon}{r-1}}=: \Lambda(t)
$$

which implies

$$
f_{t}(\theta) \leqslant e^{-r \theta \Lambda(t)}+n \eta
$$

Now, setting $\theta=\delta_{\varepsilon} \log ^{-2} n$, using the bound $\mathbf{1}_{\{X>a\}} \leqslant X / a$,

$$
\begin{aligned}
\operatorname{Pr}\{\Phi(t)<\Lambda(t)\} & \leqslant \operatorname{Pr}\{\overline{\mathcal{X}(t)}\}+\operatorname{Pr}\{\Phi(t)<\Lambda(t), \mathcal{X}(t)\} \\
& \leqslant \eta+\mathbb{E}\left(\mathbf{1}_{\left\{e^{-\theta \Phi(t)}>e^{-\theta \Lambda(t)}\right\}} \mathbf{1}_{t}\right) \\
& \leqslant \eta+e^{\theta \Lambda(t)} f_{t}(\theta) \\
& =O\left(n e^{\theta \Lambda(t)} \eta\right)+e^{-\theta(r-1) \Lambda(t)} .
\end{aligned}
$$


We bound the two terms in (22) separately. Firstly,

$$
n e^{\theta \Lambda(t)} \eta=n \exp \left\{\frac{\delta_{\varepsilon}}{\log ^{2} n} n \delta^{\frac{1+\varepsilon}{r-1}}-\frac{\varepsilon_{1} n \delta_{\varepsilon}}{\log n}\right\} \leqslant n \exp \left\{n \delta_{\varepsilon}\left(\frac{\delta_{1}^{\frac{1+\varepsilon}{r-1}}}{\log ^{2} n}-\frac{\varepsilon_{1}}{\log n}\right)\right\}
$$

and as $\delta=o(1)$ and $n \delta_{\varepsilon} / \log n=\widetilde{\Omega}\left(n^{\frac{1+\varepsilon}{r+\varepsilon}}\right)$, we have $n e^{\theta \Lambda(t)} \eta=o\left(n^{-1}\right)$. Secondly, for $\delta \geqslant \delta_{\varepsilon}=\left(n^{-1} \log ^{4} n\right)^{(r-1) /(r+\varepsilon)}$,

$$
e^{-\theta(r-1) \Lambda(t)}=\exp \left\{-(r-1) \frac{\delta_{\varepsilon}}{\log ^{2} n} n \delta^{\frac{1+\varepsilon}{r-1}}\right\} \leqslant \exp \left\{-\frac{n \delta_{\varepsilon}^{\frac{r+\varepsilon}{r-1}}}{\log ^{2} n}\right\}=e^{-\log ^{2} n}
$$

so combining (22), (23) and (24), we conclude

$$
\operatorname{Pr}\left\{\exists t_{1} \leqslant t \leqslant t_{\varepsilon}: \Phi(t)<\Lambda(t)\right\} \leqslant n\left(o\left(n^{-1}\right)+O\left(e^{-\log ^{2} n}\right)\right)=o(1) .
$$

\section{References}

[1] Miklós Ajtai, János Komlós and Endre Szemerédi, Deterministic Simulation in LOGSPACE, Proceedings of the 19th annual ACM Symposium on Theory of Computing, 1987, New York, New York, USA, pages 132-140. ACM, 1987.

[2] David Aldous and James Allen Fill. Reversible Markov chains and random walks on graphs, 2002. Unfinished monograph, recompiled 2014, available at http://www . stat . berkeley . edu/ aldous/RWG/book.html.

[3] Petra Berenbrink, Colin Cooper and Tom Friedetzky. Random walks which prefer unvisited edges: exploring high girth even degree expanders in linear time. Random Struct. Algorithms, 46(1):36-54, 2015.

[4] Béla Bollobás. A probabilistic proof of an asymptotic formula for the number of labelled regular graphs. Eur. J. Comb., 1(4):311-316, 1980.

[5] Colin Cooper, Alan M. Frieze and Tony Johansson. The cover time of a biased random walk on a random cubic graph. Proceedings of AofA 2018, 110:16:1-16:12, 2018.

[6] Colin Cooper and Alan M. Frieze. The cover time of random regular graphs. SIAM J. Discrete Math., 18(4):728-740, 2005.

[7] Colin Cooper and Alan M. Frieze. Vacant sets and vacant nets: component structures induced by a random walk. SIAM J. Discrete Math., 30(1):166-205, 2016.

[8] Joel Friedman. A proof of Alon's second-eigenvalue conjecture. In Proceedings of the Thiry-fifth Annual ACM Symposium on Theory of Computing, STOC '03, pages 720-724, New York, NY, USA, 2003. ACM.

[9] Alan M. Frieze and Michał Karoński. Introduction to Random Graphs. Cambridge University Press, Cambridge, UK, 2015. 
[10] Tal Orenshtein and Igor Shinkar. Greedy random walk. Combinatorics, Probability \& Computing, 23(2):269-289, 2014.

[11] Alistar Sinclair and Mark Jerrum. Approximate counting, uniform generation and rapidly mixing Markov chains. Inf. Comput., 82(1):93-133, 1989.

\section{A From expectation to w.h.p.}

We have calculated the expected partial edge cover time of the random regular graph, $\mathbb{E}(C(t))$, with the expectation taken over the full probability space of random wak and random graph. Let $\mathcal{G}=\mathcal{G}_{r, n}$ be the class of $r$-regular (multi-)graphs on $n$ vertices. In this section we show that if $G \in \mathcal{G}$ is chosen u.a.r., then w.h.p., $\mathbb{E}_{G}(C(t)) \sim \mathbb{E}(C(t))$, where $\mathbb{E}_{G}(C(t))$ is the expectation with $G$ fixed.

The following lemma is a direct generalization of the corresponding lemma for $r=3$ found in [5]. We remark that the class of $r$-regular multigraphs $\mathcal{G}_{n, r}$ may be replaced in the statement by the class of $r$-regular simple graphs $\mathcal{G}_{n, r}^{\prime}$, as $\left|\mathcal{G}_{n, r}^{\prime}\right| \geqslant c_{r}\left|\mathcal{G}_{n, r}\right|$ for some $c_{r}>0$ independent of $n$, see e.g. [9, Theorem 10.3].

Recall that $\delta_{2}=\log ^{-2} n$ and $t_{2}=\left(1-\delta_{2}\right) \frac{r n}{2}$.

Lemma 23. Let $t>t_{2}$ and $s \geqslant\left(1-\log ^{-r} n\right) n$. If $G \in \mathcal{G}_{n, r}$ is chosen uniformly at random, then with high probability,

$$
\begin{aligned}
\mathbb{E}_{G}(C(t)) & =\frac{r \pm \varepsilon}{2(r-2)} n \log \left(\frac{r n}{r n-2 t+1}\right), \\
\mathbb{E}_{G}\left(C_{V}(s)\right) & =\frac{1 \pm \varepsilon}{r-2} n \log \left(\frac{n}{n-s+1}\right) .
\end{aligned}
$$

Proof. Fix some $t>t_{2}$, and some constant $\varepsilon>0$ and some $\omega$ tending to infinity arbitrarily slowly with $\omega$. Let $\Delta(s)=C(s+1)-C(s)$. We define the following subfamilies of $\mathcal{G}=\mathcal{G}_{n, r}$.

$$
\begin{aligned}
\mathcal{H} & =\left\{G \in \mathcal{G}:\left|\sum_{s=t_{2}}^{t-1}\left(\mathbb{E}_{G}(\Delta(s))-\frac{1}{r-2} \frac{r n}{r n-2 s}\right)\right| \geqslant \varepsilon n \log \left(\frac{r n}{r n-2 t+1}\right)\right\}, \\
\mathcal{J} & =\left\{G \in \mathcal{G}: \mathbb{E}_{G}\left(C\left(t_{2}\right)\right) \geqslant \frac{n \log n}{\omega}\right\}, \\
\mathcal{L} & =\{G \in \mathcal{G}: \lambda(G)>0.99\} .
\end{aligned}
$$

We will show that the union of these families has size $o(|\mathcal{G}|)$. In particular, almost all $G \in \mathcal{G}$ are in $\overline{\mathcal{H}} \cap \overline{\mathcal{J}}$, which implies that $G$ satisfies $(25)$.

It follows from Lemma 3 that $|\mathcal{L}| /|\mathcal{G}|=o(1)$. By Lemma 13 we have $\mathbb{E}\left(C\left(t_{1}\right)\right)=$ $o(n \log n)$. By Lemma 14,

$$
\mathbb{E}\left(C\left(t_{2}\right)\right)-\mathbb{E}\left(C\left(t_{1}\right)\right)=O\left(\sum_{t=t_{1}}^{t_{2}} \frac{r n}{r n-2 t}\right)=O\left(n \log \left(\frac{\delta_{1}}{\delta_{2}}\right)\right)=o(n \log n) .
$$


We insist that $\omega$ is small enough so that $\mathbb{E}\left(C\left(t_{2}\right)\right) \leqslant \omega^{-2} n \log n$. Then

$$
\frac{n \log n}{\omega^{2}} \geqslant \mathbb{E}\left(C\left(t_{2}\right)\right)=\frac{1}{|\mathcal{G}|} \sum_{G \in \mathcal{G}} \mathbb{E}_{G}\left(C\left(t_{2}\right)\right) \geqslant \frac{|\mathcal{J}|}{|\mathcal{G}|} \frac{n \log n}{\omega},
$$

from which we conclude that $|\mathcal{J}| \leqslant \omega^{-1}|\mathcal{G}|$. Define $\mathcal{G}^{\prime}=\mathcal{G} \backslash(\mathcal{J} \cup \mathcal{K} \cup \mathcal{L})$ and $\mathcal{H}^{\prime}=\mathcal{H} \cap \mathcal{G}^{\prime}$. We will show that $\left|\mathcal{H}^{\prime}\right| /\left|\mathcal{G}^{\prime}\right|=o(1)$, which implies $|\mathcal{H}| /|\mathcal{G}|=o(1)$.

For $G \in \mathcal{G}^{\prime}$, let $T_{G}$ denote the set of times $s$ where $\mathbb{E}_{G}(\Delta(s))$ deviates from $\mathbb{E}(\Delta(s))$, by

$$
T_{G}=\left\{t_{1} \leqslant s<t:\left|\mathbb{E}_{G}(\Delta(s))-\frac{1}{r-2} \frac{r n}{r n-2 s}\right| \geqslant \frac{\varepsilon n}{r n-2 s}\right\} .
$$

We define a family $\mathcal{F}^{\prime} \subseteq \mathcal{G}^{\prime}$ of graphs $G$ where the contribution from $T_{G}$ is large, viz.

$$
\mathcal{F}^{\prime}=\left\{G \in \mathcal{G}^{\prime}: \sum_{s \in T_{G}} \frac{n}{r n-2 s} \geqslant \frac{n}{\omega} \log \left(\frac{r n}{r n-2 t+1}\right)\right\} .
$$

As $|\bar{X}(s)| \geqslant(r n-2 s) / r$ for all $s$, if $G \in \mathcal{G}^{\prime}$ then (letting $W(s)$ denote the history of the walk up to time $s$ ),

$$
\mathbb{E}_{G}(\Delta(s) \mid W(s)) \leqslant \frac{1}{1-\lambda(G)} \frac{n}{|\bar{X}(s)|} \leqslant \frac{100 r n}{r n-2 s} .
$$

So, if $G \in \mathcal{G}^{\prime} \backslash \mathcal{F}^{\prime}$,

$$
\begin{aligned}
& \left|\sum_{s=t_{1}}^{t-1}\left(\mathbb{E}_{G}(\Delta(s))-\frac{1}{r-2} \frac{r n}{r n-2 s}\right)\right| \\
\leqslant & \varepsilon \sum_{s \notin T_{G}} \frac{n}{r n-2 s}+\sum_{s \in T_{G}}\left|\mathbb{E}_{G}(\Delta(s))-\frac{1}{r-2} \frac{r n}{r n-2 s}\right| \\
\leqslant & \varepsilon \sum_{s=t_{1}}^{t-1} \frac{n}{r n-2 s}+\sum_{s \in T_{G}}\left(\frac{100 r n}{r n-2 s}+\frac{1}{r-2} \frac{r n}{r n-2 s}\right) \\
\leqslant & \left(\frac{\varepsilon}{2}+O\left(\frac{1}{\omega}\right)\right) n \log \left(\frac{r n}{r n-2 t+1}\right) .
\end{aligned}
$$

It follows that $\mathcal{H}^{\prime} \subseteq \mathcal{F}^{\prime}$, and it remains to show that $\left|\mathcal{F}^{\prime}\right| /\left|\mathcal{G}^{\prime}\right|=o(1)$. For $t_{1} \leqslant s<t$ let $\mathcal{F}_{s}^{\prime} \subseteq \mathcal{G}^{\prime}$ denote the set of graphs $G$ with $s \in T_{G}$.

Recall the definition of $\mathcal{E}(s)$ from Section 6.2: we say that $\mathcal{E}(s)$ holds if $X_{1}^{g}(s)=$ $(r n-2 s)(1-o(1))$ and $\bar{X}(s)$ is a root set of order $\omega$. Repeating the calculation of $(14)$ with $\varepsilon / 2$ in place of $\varepsilon$, and conditioning on $G$, we have

$$
\begin{aligned}
\mathbb{E}_{G}(\Delta(s))=\left(\frac{r}{r-2} \pm \frac{\varepsilon}{2}\right) \frac{n}{r n-2 s} \operatorname{Pr}\{\mathcal{E}(s) \mid & G\} \\
& +O\left(\frac{n}{r n-2 s} \operatorname{Pr}\{\overline{\mathcal{E}(s)} \mid G\}+\log n\right) .
\end{aligned}
$$


Let $s \geqslant t_{2}$ so that the $\log n$ term is insignificant. If $G \in \mathcal{F}_{s}^{\prime}$, so that $s \in T_{G}$, then we must have $\operatorname{Pr}\{\overline{\mathcal{E}(s)} \mid G\}=\Omega(1)$. But as shown in Section 6.2 we have $\operatorname{Pr}\{\mathcal{E}(s)\}=1-o(1)$, so almost all graphs $G$ have $\operatorname{Pr}\{\mathcal{E}(s) \mid G\}=1-o(1)$, and it follows that $\left|\mathcal{F}_{s}^{\prime}\right| /\left|\mathcal{G}^{\prime}\right|=o(1)$. Suppose $\omega$ is small enough that $\left|\mathcal{F}_{s}^{\prime}\right| /\left|\mathcal{G}^{\prime}\right| \leqslant \omega^{-2}$ for all $s$. Then

$$
\frac{1}{\left|\mathcal{G}^{\prime}\right|} \sum_{G \in \mathcal{G}^{\prime}} \sum_{\substack{s \in T_{G} \\ s \leqslant t}} \frac{n}{r n-2 s}=\sum_{s=1}^{t} \frac{\left|\mathcal{F}_{s}^{\prime}\right|}{\left|\mathcal{G}^{\prime}\right|} \frac{n}{r n-2 s} \leqslant \frac{1}{\omega^{2}} n \log \left(\frac{r n}{r n-2 t+1}\right) .
$$

But by definition of $\mathcal{F}^{\prime}$,

$$
\frac{1}{\left|\mathcal{G}^{\prime}\right|} \sum_{G \in \mathcal{G}^{\prime}} \sum_{s \in T_{G}} \frac{n}{r n-2 s} \geqslant \frac{\left|\mathcal{F}^{\prime}\right|}{\left|\mathcal{G}^{\prime}\right|} \frac{1}{\omega} n \log \left(\frac{r n}{r n-2 t+1}\right) .
$$

We conclude that $\left|\mathcal{F}^{\prime}\right| /\left|\mathcal{G}^{\prime}\right| \leqslant \omega^{-1}$, which finishes the proof for the edge cover time.

The vertex cover time statement follows from the edge cover time statement in the same manner as the proof of Lemma 17. 